\title{
A variable-fidelity aerodynamic model using proper orthogonal decomposition
}

\author{
M.J. Mifsud, D.G. MacManus and S.T. Shaw \\ School of Engineering, Cranfield University, Bedfordshire, United Kingdom, MK43 0AL
}

\begin{abstract}
A variable-fidelity aerodynamic model based on proper orthogonal decomposition (POD) of an ensemble of computational fluid dynamics (CFD) solutions at different parameters is presented in this article. The ensemble of CFD solutions consists of two subsets of numerical solutions or snapshots computed at two different nominal orders of accuracy or discretization. These two subsets are referred to as the low- and high-fidelity solutions or data, whereby the low-fidelity corresponds with computations made at the lower nominal order of accuracy or coarser discretization. In this model, the relatively inexpensive low-fidelity data and the more accurate but expensive high-fidelity data are considered altogether to devise an efficient prediction methodology involving as few high-fidelity analyses as possible, while obtaining the desired level of detail and accuracy. The POD of this set of variable-fidelity data produces an optimal linear set of orthogonal basis vectors that best describe the ensemble of numerical solutions altogether. These solutions are projected onto this set of basis vectors to provide a finite set of scalar coefficients that represent either the low-fidelity or high-fidelity solutions. Subsequently, a global response surface is constructed through this set of projection coefficients for each basis vector, which allows predictions to be made at parameter combinations not in the original set of observations. This approach is used to predict supersonic flow over a slender configuration using Navier-Stokes solutions that are computed at two different levels of nominal accuracy as the low- and high-fidelity solutions. The numerical examples show that the proposed model is efficient and sufficiently accurate.
\end{abstract}

Keywords: variable-fidelity modelling, surrogate modelling, proper orthogonal decomposition, singular value decomposition, response surface, radial basis function 


\section{Nomenclature}

$\alpha_{i j} \quad$ is the $\mathrm{i}$-th scalar or projection coefficient of the $\mathrm{j}$-th POD mode

$\Phi_{i j} \quad$ is the i-th component of the j-th POD basis mode

$m \quad$ is the number of realizations or snapshots and POD modes

$n \quad$ is the number of grid points in a mesh

a is a solution vector of primitive or conservative variables

$f(x) \quad$ computationally expensive analysis

$\tilde{f}(x) \quad$ approximation to $f(x)$

$r \quad$ radial distance from a given centre point

$\Psi(r) \quad$ a typical radial basis function

$p(x) \quad$ a polynomial of one degree less than the radial basis function $\Psi(r)$

$\alpha \quad$ angle of incidence or angle of attack

D mid-body diameter

M Mach number

$\mathrm{C}_{\mathrm{x}} \quad$ component of the aerodynamic force along the axis of the body

$\mathrm{C}_{\mathrm{z}} \quad$ component of the aerodynamic force normal to the axis of the body

$\mathrm{C}_{\mathrm{m}} \quad$ pitching moment acting on the body

$\mathrm{X}_{\mathrm{cp}} \quad$ centre of pressure coordinate along the body axis 


\subsection{Introduction}

A significant disadvantage of surrogate modelling based on proper orthogonal decomposition (POD) of an ensemble of CFD solutions is the up-front cost which is necessary to generate the dataset of observations. From this dataset, an adequately rich set of orthogonal basis vectors are constructed and subsequently used in the development of the surrogate model. Consequently, a method that reduces the time in generating this training dataset without a significant loss of accuracy is of interest. In an effort to limit this initial computational encumbrance, a variablefidelity model that incorporates the POD of an ensemble of CFD solutions at two different levels of fidelity is proposed in this paper so as to manage and control the up-front computational cost. Furthermore, this model provides a means for fusing computational data of variable-fidelity while yielding solutions for the complete flow-field.

The variable-fidelity modelling concept was employed by various researchers such as Haftka [1], Hutchinson et al. [2], Kaufmann et al. [3] and Alexandrov et al. [4][5][6][7][8] for solving design optimisation problems. In general, variable-fidelity models (sometimes these are referred to as multi-fidelity models) combine inexpensive low-fidelity analyses with more accurate but expensive high-fidelity solutions. This combination is sought, as the evaluation of high-fidelity flow simulations can be computationally expensive and therefore it is of great interest to devise methodologies, most especially in design and analysis activities, that involve as few high-fidelity analyses as possible. The variable-fidelity modelling strategy generally uses multi-dimensional response surface technology to model the different fidelities. In this concept, a model of lowerfidelity such as a coarser discretization, relaxed convergence tolerances and omitted physics are used to reduce the number of analyses using a high-fidelity model. As a result, the original complex and time consuming problem is mitigated and therefore a considerable reduction in the computational demand is achieved.

There are various strategies for reducing the number of high-fidelity analyses. Haftka [1] introduced the notion of employing a linearly varying scaling factor between models of variablefidelity. In this strategy, a high number of points are selected for the relatively inexpensive lowfidelity analyses and from these points a subset is chosen for high-fidelity analyses. The lowfidelity results are used to fit a response surface while the high-fidelity analyses are used to fit a 
linear correction response surface. For a common design point between the two models, the ratio of the responses is evaluated and used for establishing the variable-fidelity approximation. Subsequently, the concept of correction response surfaces was applied by others such as Hutchinson et al. [2]. Another strategy presented by Kaufmann et al. [3], entails the use of lowfidelity models to reduce the region in the design space and once this is reduced, a high-fidelity response surface is constructed over this reduced space.

A different strategy, primarily of interest in optimisation problems, uses a model of low-fidelity to conduct the optimization. Then occasionally and systematically information from the highfidelity model is used to check and recalibrate the designs generated. In order to manage the approximations, various ways to decide when the fidelity is increased or decreased were suggested [4][6][7][8]. An approach related to the one proposed by Haftka uses an additive correction method in which the difference between low- and high-fidelity models is evaluated. This correction is subsequently added to the low-fidelity response surface. A comparison between multiplicative and additive correction response surfaces was conducted by Toropov and Markine [9], who suggested that the multiplicative correction leads to better approximations. Another possibility is to use low-fidelity models to identify unimportant response surface terms or to identify insignificant variables that reduce the problem dimensionality as shown by Knill et al. [10].

An autoregressive co-kriging approach suggested by Kennedy and O'Hagan [11] was utilized by Huang et al. [12] and Forrester et al. [13]. The method has the advantage that it is applicable to more than two fidelity models. Leary et al. [14] presented a knowledge-based variable-fidelity approach, where low-fidelity data are dealt with as apriori knowledge in the training process of artificial neural networks and Kriging interpolation. A space-mapping model between lowfidelity and high-fidelity data using POD was suggested by Robinson et al. [15] [16]. This model is based on the gappy POD method for the reconstruction of incomplete datasets developed by Everson and Sirovich [17]. A completely different approach, which utilises radial basis functions to fuse experimental and computational integrated data (ie. aerodynamic coefficients) for a missile configuration, was proposed by Reisenthel et al. [18] and to fuse experimental and computational surface pressure data for an aircraft wing by Rendall and Allen [19]. 
In this article, a surrogate model based on POD of an ensemble of CFD snapshots at two different fidelities and at different parameters' value is coupled together with the variable-fidelity model suggested by Reisenthel et al. [18]. While Reisenthel's model can be applied over individual or integrated properties at different fidelities, the advantage of the model presented in this article is that it can be applied over the whole solution domain generated by CFD simulations. Consequently, the model produces a solution similar to that obtained from a CFD calculation. This model was originally presented by Mifsud [20].

The layout of the paper is as follows. In Section 2, a description of the various numerical methods employed is presented. In particular, a description of POD and a detailed description of the variable-fidelity model based on POD are made. In Section 3, the results obtained from the variable-fidelity model for a cone-cylinder-flare body are presented together with some recommendations in its use. In Section 4, a conclusion is drawn.

\subsection{Numerical Methods}

In this section a brief description of the numerical methods employed is presented. This includes the CFD tool, the POD methodology as well as the variable-fidelity model.

\subsection{CFD Model}

The CFD analysis tool used to generate the snapshots in this work is the IMPNS flow solver [21] [22]. The IMPNS software has been developed to provide a practical flow solver for problems in high-speed air vehicle aerodynamics and includes algorithms for the solution of the Euler, thinlayer or parabolized Navier-Stokes equations together with a range of turbulence closures. IMPNS has been used extensively to study the aerodynamics of high-speed air vehicle configurations with remarkable success. Further details of its development and application can be found in references [23] to [29]. 


\subsection{Surrogate modelling based on proper orthogonal decomposition}

The surrogate model presented in this article comprises three main elements: a dimensionality reduction method that is used to determine the dominant characteristics of a set of discrete observations at different parameters' value, a response surface representation of the scalar coefficients obtainable from the dimensionality reduction model and the variable-fidelity model. These three elements of the aerodynamic model are described in the following sections.

\subsubsection{Proper Orthogonal Decomposition}

In fluid mechanics, the POD was first introduced by Lumley [30] in the context of stochastic turbulence. The same procedure has been widely used in other disciplines and is commonly referred to as the Karhunen-Loéve expansion and the principal component analysis. POD is also very closely related to the singular value decomposition. The POD provides a basis for the modal decomposition of a system of functions, usually data obtained from either experiments or numerical simulations. The resulting basis functions are called proper orthogonal modes and are the best possible uncorrelated and data-dependent linear set of bases functions that describe the initial observations.

The POD method has been used extensively in the fields of random variables, image processing, data compression and system controls. In the field of fluid dynamics, it has been used in unsteady flow problems such as aero-elasticity and stochastic turbulence to capture the temporal variation of the flow. It was also used in steady flow problems to capture parametric variations. For example, Epureanu et al. [31] employed POD to develop reduced-order models for potential flow in turbo-machinery with sampling performed over a range of inter-blade phase angles and time. LeGresley and Alonso [32] used POD to develop surrogate models of a 2D Euler flow solver for design optimization purposes in which the POD modes spanned a range of aerofoil geometries. Bui-Thanh et al. [33] employed the POD method, together with an interpolation

procedure, to predict the pressure flow-field over an aerofoil for varying inflow Mach number and angle of attack while Mifsud et al. [34] presented a similar methodology which was applied to a high-speed aerodynamics problem in the supersonic flow regime. 
The POD procedure is usually described using the calculus of variations applied to a multidimensional spatio-temporal dataset. In such problems, the dataset is produced from solution vectors obtained at a particular instant of time. In this paper, the POD is described for steady flow problems in terms of the singular value decomposition (SVD). Although these two approaches are equivalent in a descritized context, the SVD approach is preferred as it is more straightforward.

Let $\mathbf{A} \in \mathbf{R}^{m \times n}$ denote the matrix whose rows are the snapshots with data centred about the origin. Each snapshot is constructed by placing in order the solution at each grid point in the CFD domain for the complete grid. This order can be determined arbitrarily, but it must be consistent throughout the whole set of snapshots.

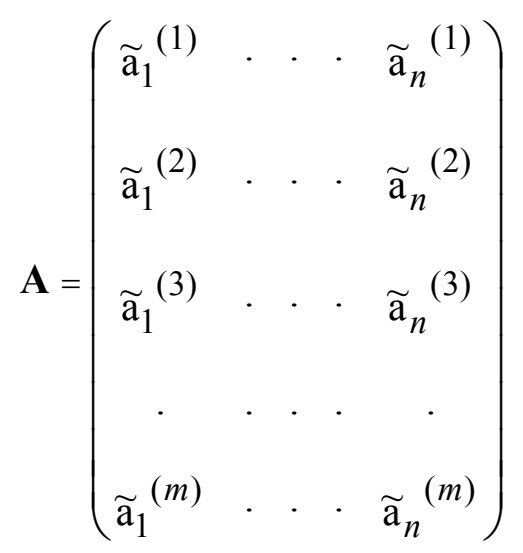

Eq. (1)

The SVD of $\mathbf{A}$ can be written as

$$
\mathbf{A}=\mathbf{U} \boldsymbol{\Sigma} \mathbf{V}^{\mathbf{T}}
$$

where $\mathbf{U} \in \mathbf{R}^{m \times m}$ and $\mathbf{V} \in \mathbf{R}^{n \times n}$ are orthogonal matrices. These matrices are the left and right singular vectors respectively. $\boldsymbol{\Sigma} \in \mathbf{R}^{m \times n}$ is a diagonal matrix whose diagonal elements consist of $q=\min (m, n)$ non-negative numbers $\sigma_{i}$ arranged in decreasing order, that is, $\sigma_{1} \geq \sigma_{2} \geq \sigma_{3} \geq \ldots . \sigma_{q}$ where $\sigma_{i}$ are referred to as the singular values of $\mathbf{A}$. Since $\boldsymbol{\Sigma}$ is a diagonal $m \times n$ matrix, then the above matrix equation can be written in reduced or thin form as 
follows if we assume that $m<n$, where the matrices $\boldsymbol{\Sigma} \in \mathbf{R}^{m \times m}$ and $\mathbf{V}^{\mathbf{T}} \in \mathbf{R}^{m \times n}$ are reduced in size. The columns of $\mathbf{V}$ and hence the rows of $\mathbf{V}^{\mathbf{T}}$ are the proper orthogonal modes of the system. These basis vectors are orthonormal. Writing the product of $\mathbf{U}$ and $\boldsymbol{\Sigma}$ as a matrix $\left[\alpha_{i j}\right]$, Eq. (2) can be represented by

$$
\widetilde{\mathrm{a}}_{k}^{(i)}=\sum_{j=1}^{m} \alpha_{i j} \mathrm{v}_{j k}
$$

The scalar coefficients $\alpha_{i j}$ are also referred to as projection coefficients because these are obtained by projecting the solution onto the basis vectors. A complete reconstruction of the snapshots can be obtained from

$\mathbf{a}=\langle\mathbf{a}\rangle+\widetilde{\mathbf{a}}$

where $\langle\mathbf{a}\rangle$ is the mean vector by which the snapshots were centred. Now, a may represent a vector of scalar functions such as the primitive or conservative variables and therefore the method described can be applied to each variable in turn to form a distinct basis for each variable. However, an improvement in the ability of the basis to represent the system may be achieved by considering not only how the individual variables vary from one snapshot to another but also how variables change relative to one another. Hence a is developed from state variable vectors consisting of all the primitive or conservative variables [32]. In this case, the POD modes are sensitive to the scaling of the flow variables as these are in different units and have significantly varying magnitudes. Consequently, appropriate scaling factors are necessary for each fluctuating flow variable which makes their magnitude of the same order [35].

When a problem is represented by an appropriate number of snapshots from which a suitably rich set of basis vectors is available, the singular values rapidly become small and a few basis vectors are adequate to reconstruct and approximate the snapshots. Assuming that $p$ modes which correspond to the largest $p$ singular values are dominant, then the energy E or variance in the data captured by the first $p$ modes can be computed as 
$\mathrm{E}(p)=\frac{\sum_{i=1}^{i=p} \sigma_{i}^{2}}{\sum_{i=1}^{i=m} \sigma_{i}^{2}}$

If this energy is sufficiently high enough, say over $99.99 \%$ of the total energy, then $p$ modes are adequate to capture the principal features and approximately reconstruct the dataset. Thus, a reduced subspace is formed which is only spanned by $p$ modes.

\subsubsection{Constructing the response surface}

The use of surrogate models based upon proper orthogonal decomposition for prediction requires the fitting of a response surface through the projection coefficients, $\alpha_{i}$. If $\alpha_{i}$ varies as a smooth function with the change in parameters, then a surrogate model may be used to determine the POD projection coefficients at intermediate parametric values not included in the original data ensemble. The predicted solution vector $\mathbf{a}^{(\beta)}$ for any variable $\beta$ within the parametric space is given by,

$\mathbf{a}^{(\beta)}=\langle\mathbf{a}\rangle+\sum_{i=1}^{p^{\prime}} \alpha_{i}^{\beta} \mathbf{v}_{i}$

where $p^{\prime}$ is normally greater than $p$ and the weighting coefficients $\alpha_{i}^{\beta}$ are found by mathematical modelling. In this work, radial basis functions that produce an interpolative fit through all of the sample points were considered.

\subsubsection{Radial Basis Functions}

A radial basis function (RBF) [36] is a real-valued function whose value depends on the Euclidean distance from some point called a centre. Radial basis functions are typically used to build up a function approximation of the form 
$\widetilde{f}(x)=p(x)+\sum_{i=1}^{N} w_{i} \Psi\left(\left\|x-x_{i}\right\|\right)$

where the approximating function $\widetilde{f}(x)$ is represented as a sum of $N$ radial basis functions $\Psi$, each associated with a different centre $x_{i}$ and weighted by an appropriate coefficient $w_{i}$. The variable $p(x)$ is a polynomial of one degree less than the RBF and is included to ensure a unique solution for the weight vector. Thus, an RBF is a weighted sum of translations of a radially symmetric basis function augmented by a polynomial term. In particular RBFs are suitable for interpolating scattered data and hence do not require the data to lie on any sort of regular grid for most types.

Typical radial basis functions are

$$
\text { Gaussian } \Psi(r)=e^{-\frac{r^{2}}{\theta}}
$$$$
\text { multi-quadric } \Psi(r)=\sqrt{1+\frac{r^{2}}{\theta}} \quad[37][38] \text {, }
$$

(iii) polyharmonics such as the triharmonic $\Psi(r)=r^{3}$ and

(iv) thin plate splines $\Psi(r)=r^{2} \ln r$

The constant $\theta$ in (i) and (ii) is called the shape parameter. The RBF interpolant $\widetilde{f}(x)$ is defined by the coefficients of the polynomial $p(x)$ and the weights $w_{i}$. Since this produces an underdetermined system, the orthogonality condition

$$
\sum_{j=1}^{N} w_{j} p\left(x_{j}\right)=0
$$

is further imposed on the coefficients $\boldsymbol{w}=\left(w_{1}, \ldots \ldots, w_{N}\right)$.

Let $P=\left(p_{1}, \ldots . ., p_{l}\right)$ be a basis for the polynomial and let $\boldsymbol{c}=\left(c_{1}, \ldots \ldots \ldots, c_{l}\right)$ be the coefficients that give $P$ in terms of this basis. Then Eq. (7) and Eq. (8) may be written in matrix form as 
$\left(\begin{array}{cc}\boldsymbol{\Psi} & \boldsymbol{P} \\ \boldsymbol{P}^{\mathrm{T}} & 0\end{array}\right)\left(\begin{array}{l}w \\ c\end{array}\right)=\left(\begin{array}{l}f \\ 0\end{array}\right)$

where $\boldsymbol{\Psi}=\left(\begin{array}{cccc}\psi(0) & \psi\left(\left\|x_{2}-x_{1}\right\|\right) & . . & \psi\left(\left\|x_{N}-x_{1}\right\|\right) \\ \psi\left(\left\|x_{1}-x_{2}\right\|\right) & \psi(0) & . . & \psi\left(\left\|x_{N}-x_{2}\right\|\right) \\ : & : & : & : \\ \psi\left(\left\|x_{1}-x_{N}\right\|\right) & \psi\left(\left\|x_{2}-x_{N}\right\|\right) & . . & \psi(0)\end{array}\right)$

and $\quad P_{i, j}=p_{j}\left(x_{i}\right), \quad i=1, \ldots \ldots . . N, j=1, \ldots \ldots \ldots . .$. . Solving Eq. (9) determines $\boldsymbol{c}$ and $\boldsymbol{w}$, hence $\widetilde{f}(x)$.

\subsubsection{The variable-fidelity model based on POD}

Assuming that the low-fidelity and the high-fidelity solutions significantly correlate with one another, a variable-fidelity model based on POD can possibly be set up in a number of ways. One possible way is to conduct the POD analysis on the high- and low-fidelity data separately and then to construct a response surface to model the discrete projection coefficients of the corresponding modes at low- and high-fidelity separately. However, this method may run into the problem that the low-fidelity and the high-fidelity POD modes are not directionally aligned with one another and hence it would not be straightforward to relate the low- and high-fidelity modes together. Moreover, this method would require a considerable number of high-fidelity snapshots, which is not a desirable characteristic. Hence, in order to circumvent these problems, a model is proposed in this work in which the POD analysis is conducted on the variable-fidelity dataset altogether. Generally, this methodology requires either a common computational grid between the low- and high- fidelity solutions or else that the low-fidelity solutions are mapped onto the high-fidelity grid by some interpolation technique.

Considering an ordered ensemble of variable-fidelity data $\mathbf{A}$, where $\mathbf{A} \in \mathbf{R}^{m \times n}$, is obtained from the solution vectors of low- and high-fidelity computations at various parameters' values. The total number of realizations or parameters combination $m=m_{1}+m_{2}$, where $m_{1}$ is the number of snapshots obtained from the high-fidelity computations and $m_{2}$ is the number of 
snapshots obtained from the low-fidelity computations. It is assumed that $m_{1}<<m_{2}$ and $n$ is the number of sample points over which the computational domain is sampled.

Let $\mathbf{A} \in \mathbf{R}^{m \times n}$ denote the matrix whose rows are the centred snapshots where the primed entries denote the high-fidelity solution vectors, from row 1 to row $m_{1}$. The non-primed entries represent the low-fidelity solution vectors, from row $m_{1}+1$ to $m$ where $m=m_{1}+m_{2}$. In this methodology, the parameters combination between the low- and high-fidelity solutions can be different and it is not necessary to have common snapshots between the variable-fidelities, although these can assist to establish apriori whether there exists a strong or weak correlation between the low- and high-fidelity solutions.

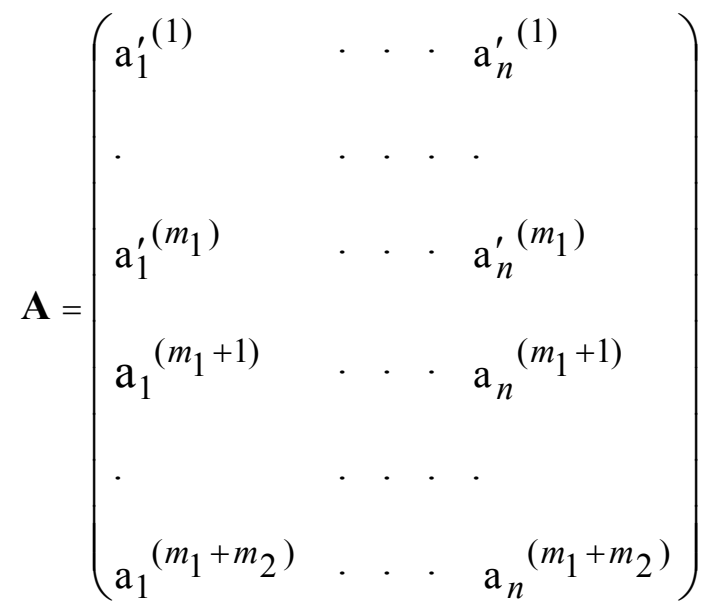

The SVD of $\mathbf{A}$ which is equal to $\mathbf{U} \boldsymbol{\Sigma} \mathbf{V}^{\mathbf{T}}$ can be written in reduced form as 


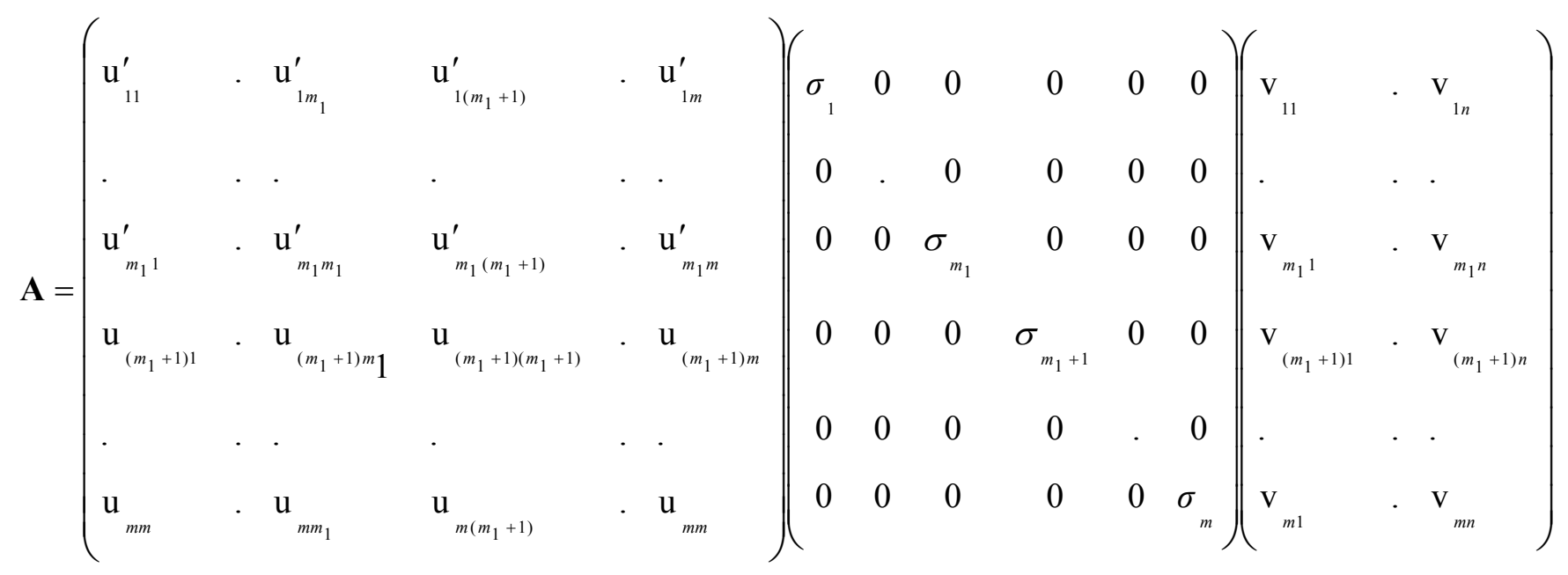

The rows of $\mathbf{V}^{\mathbf{T}}$ are the proper orthogonal modes of the system. Multiplying the first two matrices on the RHS to obtain the projection coefficients matrix $\left[\alpha_{i j}\right]$

$\mathbf{A}=\left(\begin{array}{llllll}\alpha_{11}^{\prime} & \cdot & \alpha_{1 m_{1}}^{\prime} & \alpha_{1\left(m_{1}+1\right)}^{\prime} & \cdot & \alpha_{1 m}^{\prime} \\ \cdot & \cdot & \cdot & \cdot & \cdot & \cdot \\ \alpha_{m_{1} 1}^{\prime} & \cdot & \alpha_{m_{1} m_{1}}^{\prime} & \alpha_{m_{1}\left(m_{1}+1\right)}^{\prime} & \cdot & \alpha_{m_{1} m}^{\prime} \\ \alpha_{\left(m_{1}+1\right) 1} & \cdot & \alpha_{\left(m_{1}+1\right) m_{1}} & \alpha_{\left(m_{1}+1\right)\left(m_{1}+1\right)} & \cdot & \alpha_{\left(m_{1}+1\right) m} \\ \cdot & \cdot & \cdot & \cdot & \cdot & \cdot \\ \alpha_{m m} & \cdot & \alpha_{m m_{1}} & \alpha_{m\left(m_{1}+1\right)} & \cdot & \alpha_{m m}\end{array}\right) \cdot\left(\begin{array}{lll}\mathrm{v}_{11} & \cdot & \mathrm{v}_{1 n} \\ \cdot & \cdot & \cdot \\ \mathrm{v}_{m_{1} 1} & \cdot & \mathrm{v}_{m_{1} n} \\ \mathrm{v}_{\left(m_{1}+1\right) 1} & \cdot & \mathrm{v}_{\left(m_{1}+1\right) n} \\ \cdot & \cdot & \cdot \\ \mathrm{v}_{m 1} & \cdot & \mathrm{v}_{m n}\end{array}\right)$

From this projection coefficients matrix, the set of scalar coefficients

and

are considered altogether to develop multi-dimensional response surfaces for variable-fidelity data representation. Note that the high-fidelity response surface part is formed by the projection coefficients for while the low-fidelity response surface part is formed by the projection coefficients for $\quad$ This is achieved by the introduction of an auxiliary variable $\varepsilon \equiv x_{N+1}$ to the $N$-dimensional problem with variables $\left(x_{1}, x_{2}, \ldots \ldots, x_{N}\right)$. This auxiliary variable simply denotes whether the data is of low-fidelity $(\varepsilon=0)$ or high-fidelity $(\varepsilon=$ 1). From this, a global response surface is then computed in the $N+1$ dimensions using radial basis functions. Predictions can be made by interrogating the newly developed response surface projected along $\varepsilon=1$ at any arbitrary parameter value not in the original set of observations. The 
resulting model representation respects the accuracy of the high-fidelity data while following the features of the low-fidelity data. In this model, the introduction of the low-fidelity sub-space by making use of an additional variable, allows an interpolation-based extrapolation to be performed. In other words, an extrapolation at high-fidelity based on interpolation at low-fidelity is conducted.

Following this, the predicted solution vector $\hat{\mathbf{a}}$ is determined by

$$
\hat{\mathbf{a}}=\langle\mathbf{a}\rangle+\sum_{k=1}^{q} \alpha_{i=\text { const }, k}^{\prime} \mathbf{v}_{k, j=\text { const }}
$$

where $q<m$ and $\langle\mathbf{a}\rangle$ is the mean vector. It is important to emphasize that this model gives access to the full flow-field data.

\subsection{Results}

The variable - fidelity model based on POD and described in the preceding section was tested by modelling supersonic flow over a high-speed air vehicle configuration as a function of two different flow parameters. The two flow parameters of interest were the flight Mach number (M) and the angle of incidence $(\alpha)$. The geometry considered is a flare stabilized projectile as shown in Figure 1. It comprises a conical fore-body of length 3.56 mid-body diameters, a cylindrical centre-body of length 15.34 mid-body diameters with an aft flare which increases in diameter from one mid-body diameter to four mid-body diameters over a length of 4.24 diameters. All CFD computations were conducted using the IMPNS software on a half-body computational mesh with 26 grid points in the axial direction, 41 grid points in the radial direction and 31 grid points in the circumferential direction. No geometrical body deformations were considered.

An ensemble of CFD solutions at different values of the flow parameters and at two different levels of fidelity were generated. The two levels of data were computed using a different nominal order of accuracy in the computational scheme. This particular problem is of interest because PNS flow solvers tend to encounter difficulties when computing at high angles of incidence and at a high nominal order of accuracy, and despite these difficulties, a result would be highly 
desireable even if it is inaccurate. The variable-fidelity model can be equally applied to a problem where coarse and fine grids are considered to create the low- and high-fidelity sets of data respectively. In that case, however, a technique to map the larger number of points of the fine grid onto the coarse grid is necessary.

As stated ealier, the two flow parameters considered were the angle of incidence which was varied within the range $\left[0^{\circ}, 10^{\circ}\right]$ and the Mach number which was varied within the range $[2,6]$. The two subsets of snapshots were generated by running the IMPNS software over the entire sampling space at a constant Reynolds number of 0.4 million based on the mid-body diameter. Fully turbulent flow was considered. The low-fidelity snapshots were generated by computing nominally first-order accurate calculations, while the high-fidelity snapshots were calculated using a nominally third order accuracy in the cross-flow directions and second order in the stream-wise.

To investigate apriori the strength or weakness of the correlation between the low- and highfidelity solutions, the Pearson's product - moment correlation coefficient was calculated for the surface parameters at low- and high-fidelity. The coefficient of pressure $C_{p}$ and the surface friction coefficients $\tau_{\mathrm{x}}, \tau_{\mathrm{y}}$ and $\tau_{\mathrm{z}}$ in the $\mathrm{x}, \mathrm{y}$ and $\mathrm{z}$ directions respectively were considered. Note that the correlation coefficient could have been evaluated upon the primitive variables instead.

\subsection{Variable--fidelity correlations}

Table 1 shows the variable-fidelity correlations at Mach numbers 2 and 6. At a Mach number of 2 , the correlation coefficient between the low- and high-fidelity snapshots indicates a strong correlation in $\mathrm{C}_{\mathrm{p}}$ throughout the whole range for the angle of attack. While it shows good correlation in surface friction towards the ends of the angle of attack range, this correlation deteriorates towards the mid-range at an angle of attack of $5^{\circ}$. Table 1 also includes the $95 \%$ confidence that the correlation coefficient is between the lower and the upper limits. In addition these data provide significant evidence for the alternative hypothesis with a p-value less than 2.2 $\times 10^{-16}$. 
Also at a Mach number of 6 , the correlation coefficient indicates a strong correlation for $\mathrm{C}_{\mathrm{p}}$ throughout the whole angle of attack range. The correlation coefficient based upon the surface friction is relatively weak at $0^{\circ}$ and steadily increases towards an angle of attack of $10^{\circ}$. Moreover, these data provide significant evidence for the alternative hypothesis with a p-value less than $2.2 \times 10^{-16}$. These results indicate that reasonably good results are expected from the variable-fidelity model based on POD and this will be evident in the next two sections.

\subsection{Training the variable-fidelity model with five high-fidelity snapshots}

In this work, two problems were set-up with different training datasets. In the first problem, the training data consisted of the following two subsets:

i. For the low-fidelity dataset: 30 snapshots were generated with the Mach number varying within the range $[2,6]$ in increments of 1.0 , while the angle of incidence varying within the range $\left[0^{\circ}, 10^{\circ}\right]$ in increments of $2^{\circ}$.

ii. For the high-fidelity dataset: 5 snapshots were generated with the Mach number varying within the range $[2,6]$ in increments of 1.0 and at a constant angle of incidence of $5^{\circ}$.

As explained previously, the two dimensional problem was augmented with an auxiliary variable denoting whether the data is of low- or high- fidelity, giving rise to a three-dimensional problem ie., the two aerodynamic parameters ( $\mathrm{M}$ and $\alpha$ ) and the auxiliary variable $(\varepsilon)$. With this training data, a global response surface of the projection coefficients was generated using Gaussian radial basis functions with a shape parameter of 1.0 and this was used as a global interpolant along the three dimensions. From the interpolated projection coefficients, predictions were generated of the full three-dimensional flow field which was then used to calculate the main integral parameters of interest, the viscous axial force coefficient $C_{x}$ and the normal force coefficient $C_{z}$.

Figures 2 and 3 show the variation of the predicted viscous axial force coefficient $C_{x}$ and the normal force coefficient $\mathrm{C}_{\mathrm{z}}$ with the angle of incidence for the cone-cylinder-flare body at a Mach number of 2 and 6 respectively. The figures also include the high-order accurate solution 
(high-fidelity) for verification purposes and the first-order accurate solution (low-fidelity) to show its trend. The training data which is partially input into the model at the respective Mach number are also shown and denoted by a diamond symbol. From Figures 2 and 3 it can be noticed that the predictions are reasonably good and generally follow the trend of the low-fidelity data while passing through the high-fidelity data point used in the training dataset at an angle of attack of $5^{\circ}$. During modelling, it was observed that this effect can even be made either more pronounced or else alleviated by an appropriate scaling of the magnitude of the auxiliary variable $\varepsilon$.

The agreement between the predictions for the proposed model and the high-fidelity solutions are particularly good at a Mach number of 2 and reasonably good at a Mach number of 6 . The errors at a Mach number of 2 are within $\pm 2.0 \%$ for $\mathrm{C}_{\mathrm{x}}$ and $\pm 6.0 \%$ for $\mathrm{C}_{\mathrm{z}}$ of the high-fidelity values. At a Mach number of 6 , the errors are within $\pm 6.0 \%$ for $\mathrm{Cx}$ and $\pm 15.0 \%$ for $\mathrm{Cz}$ of the high-fidelity values. These results emphasize the importance of using low-fidelity models which incorporate the correct trends, as the biggest errors were observed in those regions where the low- and high-fidelity follow different trends and have different features. This is most especially important when a few high-fidelity data points are taken into consideration, since the reliance on the low-fidelity solutions is considerably greater. This observation was also made by Reisenthel et al. [18].

\subsection{Training the variable-fidelity model with fifteen high-fidelity snapshots}

In an effort to improve upon the results obtained from the variable-fidelity model, the training dataset was enriched by increasing the number of high-fidelity training data such that fifteen snapshots were considered together with the previous low-fidelity dataset. A full-factorial design-of-experiment was set up with Mach numbers at 2, 3, 4, 5 and 6, and an angle of attack of $2^{\circ}, 5^{\circ}$ and $8^{\circ}$. These angles of attack were chosen so that the high-fidelity sub-space partially covers the whole parametric space. Therefore, this would provide a problem where some regions would be predicted by interpolation and some others by extrapolation. This dataset of observations was used to train a three-dimensional response surface which was once again used as a global interpolant along the three-dimensions. 
The variation of the viscous axial and normal force coefficients with the angle of attack at different Mach numbers are shown in Figures 4 and 5. As expected, significant improvement is achieved when using more high-fidelity data points over a wider area of the evaluation space as is evident in the predictions at the higher Mach number (Figure 5). Furthermore, from these results at both Mach numbers of 2.0 and 6.0, it is clear that by using the variable-fidelity model based on POD, an improvement is registered at regions beyond the high-fidelity data. This is evident when comparing the results obtained from the variable-fidelity model based on POD with the prediction generated using the high-fidelity snapshots only. This latter prediction is denoted by Prediction V-S only in Figures 4 and 5. This improvement is achieved due to the introduction of the low-fidelity sub-space to the problem which allows the extrapolation of the high-fidelity sub-space based on an interpolation of the low-fidelity sub-space. In this case, the errors at a Mach number of 2 are within $\pm 1.0 \%$ for $\mathrm{C}_{\mathrm{x}}$ and $\pm 5.0 \%$ for $\mathrm{C}_{\mathrm{z}}$ of the high-fidelity values. At a Mach number of 6 , the errors are within $\pm 2.0 \%$ for $\mathrm{Cx}$ and $\pm 8.0 \%$ for $\mathrm{Cz}$ of the high-fidelity values.

Figures 6 and 7 show the projected carpet plots of the viscous axial and normal force coefficients using different methods. The sub figures 6 (a) and 7 (a) show the results from the high-order accurate calculation using the IMPNS CFD code. The sub figures (b) and (c) show the results from the variable-fidelity model based on POD using five and fifteen high-fidelity snapshots, respectively. It is clear that when using five high-fidelity snapshots (Figure 6 (b) and Figure 7 (b)), the discrepancies between the predicted and the high-order accurate CFD solutions are evident. These differences reduce substantially and tend to become insignificant when considering fifteen high-fidelity snapshots (Figure 6(c) and Figure 7(c)).

\subsection{Flow-field reconstruction using the variable-fidelity model}

In the first part of the assessment of the VFM based on POD approach, the performance was evaluated by considering the integrated properties such as the force coefficients. However, these are only a partial outcome from the proposed variable-fidelity model. In general, information on the details of the flow-field is also of interest and this is indeed provided by the VFM based on POD approach. Figures 8 and 9 show the total pressure contours along the symmetry plane and 
the body surface, while Figures 10 and 11 show the total pressure contours at the outflow and the body surface. Note that the total pressure contours were cut-off over $3225 \mathrm{~Pa}$ and below $325 \mathrm{~Pa}$ for clarity. The Figure 8 (a) and Figure 9 (a) represent the prediction from the VFM based on POD model and Figure 8 (b) and Figure 9 (b) are obtained from the full order CFD solution. These figures compare the total pressure contours for the high-fidelity CFD simulations and the model predictions with fifteen high-fidelity snapshots for a Mach number of 2.0 and at angles of incidence of $6^{\circ}$ and $9^{\circ}$. The incidence setting of $6^{\circ}$ sits within the high-fidelity training dataset while that of $9^{\circ}$ is outside. At these parametric values, reasonably accurate viscous axial and normal force coefficients and their derivatives were attained and so it is interesting to observe the flow-field predictions. In this case, the total pressure was considered since it is a sensible indication of the accuracy by which all the primitive variables are predicted. The comparisons show that the predicted total pressure contours are only slightly different from the ones calculated over certain regions of the flow-field.

\subsection{Comments and recommendations}

The model presented in this article consists of coupling together a variable-fidelity model and a reduced-order model based on POD. It is therefore expected that the model inherits both the advantages and disadvantages from these two elements. In fact, the reduced-order modelling limitations are still present within the proposed method. Also, it was observed that the model provides reasonably accurate predictions when there exists a significant correlation between the low-fidelity and the high-fidelity training data. As a consequence, the model cannot be used when there is significant difference in the flow physics between the low-fidelity and high-fidelity training data. For example, the model would not predict sensibly when considering a flow over an aerofoil in the transonic flow regime and using inviscid and viscous flow computations as the low- and high-fidelity training data respectively. The difference between the shock's strength and position of an inviscid and viscous flow are substantial. Another instance when the model fails is when modelling the flow velocity and using inviscid and viscous flow training data as the low- and high-fidelity solutions respectively. Under this particular circumstance, the boundary conditions at the wall are totally different. 
On the other hand, the model provides reasonably accurate predictions when there exists significant correlation between the low-fidelity and the high-fidelity training data and this concurs with the observations reported by Reisenthal et. al [18], where the predictions were found to closely follow the trend of the low-fidelity training data points. This characteristic is most prominent when the high-fidelity training data is sparse. However, the influence of the lowfidelity data upon the high-fidelity data reduces as the number of high-fidelity training data points is increased. A way to verify whether the model is adequately representing a particular application is by increasing in steps the number of high-fidelity training data and monitoring the discrepancies from its output. As the number of the high-fidelity training data is increased, the response from the model must tend towards some limiting value, which will be within some approximation error due to the interpolation technique.

Moreover, it is well known that surrogate models based on POD work well in an interpolation based setting. However, it is not so clear whether the POD technique works well in an extrapolation based setting unless the physics of the problem changes linearly beyond the parametric range. Therefore, by using this variable-fidelity model based on POD, a transformation of the problem is made from an extrapolation setting into an interpolation one by the introduction of the auxiliary variable, hence making it possible for the variable-fidelity model based on POD to work well. Thus, this technique may be considered also as a step towards achieving a variable-fidelity model based on POD to work successfully for a linear or non-linear problem in an extrapolation based setting.

As a general recommendation, it is advisable that the scalar coefficients are scaled between 0 and 1 for each POD mode separately before generating and interrogating the response surface, even though in this work no scaling was performed. After interrogation, the coefficients are unscaled once again and used as in Eq. (12) to generate the predicted solutions. Moreover, it is suggested that in order to establish apriori whether there exists correlation between the low- and highfidelity sets of data, a Pearson's second-moment correlation coefficient is evaluated between the high- and low-fidelity solutions at common points within the parameters' space. This together with other tests would provide apriori an indication of whether the prediction is reliable or not, after the necessary calibration. 


\subsection{Conclusions}

A variable-fidelity model based on POD is applied to model the three dimensional flow around an air vehicle travelling at high speed. In this model, a POD of an ensemble of snapshots made up of two different levels of fidelity is conducted, from which the resulting low- and high-fidelity projection coefficients along each significant POD mode are mathematically modelled using a variable-fidelity model. Subsequently, high-fidelity predictions are made for the modal coefficients at parameter values that are not available in the training dataset for each POD mode. It was observed that this model behaves like other variable-fidelity models in that the highfidelity prediction follows very closely the trend of the low-fidelity training data points. Thus, the resulting predictions depend on the low-fidelity dataset, especially when the high-fidelity training data is sparse. However, the predictions get more accurate and independent of the lowfidelity trend as the number of high-fidelity data points is increased. Potentially this method offers a reduction in the up-front cost necessary to generate the training dataset for a POD-based surrogate model. In particular, the model was used to fuse low-order and high-order accurate CFD solutions. It was demonstrated that this technique works well for this kind of problems where both the physics and the boundary conditions of the variable-fidelity data are similar. The model was sequentially applied directly on all of the primitive variables and good agreement was

found between the model predictions and the high-order accurate CFD solutions at the same parametric values. 


\section{References}

[1] Haftka, R.T., 'Combining Global and Local Approximations', AIAA Journal, Vol. 29 No. 9, pp. 1523-1525, 1991.

[2] Hutchinson, M.G., Unger, E.R., Mason, W.H., Grossmann, B., and Haftka, R.T., 'Variable-Complexity Aerodynamic Optimization of a High-Speed Civil Transport Wing', Journal of Aircraft, Vol. 31, No.1, pp110-116, 1994.

[3] Kaufmann, M., Balabanov, V., Burgee, S.L., Giunta, A.A., Grossman, B., Haftka, R.T., Mason, W.H. and Watson, L.T., 'Variable-complexity response surface approximations for wing structural weight in HSCT design', Computational Mechanics, 18 (2), 112-126, 1996.

[4] Alexandrov, N.M., 'Robustness properties of a trust region framework for managing approximations in engineering optimization', 6th AIAA/NASA/ISSMO symposium on multidisciplinary analysis and optimization, Vol.2, pp. 1056-1059, AIAA-96-4102CP.Bellevue, WA, 1996

[5] Alexandrov, N.M., Dennis, J.E., Lewis, R.M., and Torczon V., 'A trust region framework for managing the use of approximation models in optimization', NASA/CR-201745, ICASE Report N. 97-50, 1997

[6] Alexandrov, N.M., Lewis, R.M., Gumbert, C.R., Green, L.L., and Newman P.A., 'Optimization with variable-fidelity models applied to wing design', AIAA Paper 20000841,2000

[7] Alexandrov, N.M., Nielsen, E.J., Lewis, R.M., and Anderson, W.K., 'First-order model management with variable-fidelity physics applied to multi-element airfoil optimization' 8th AIAA/USAF/NASA/ISSMO symposium on multidisciplinary analysis and optimization. AIAA-2000-4886, Long Beach, CA, 2000

[8] Alexandrov, N.M., Lewis, R.M., Gumbert, C.R., Green, L.L., and Newman P.A., 'Approximation and model management in aerodynamic optimization with variable fidelity models' Journal of Aircraft, 38 (6), 1093-1101, 2001

[9] Toropov, V.V. and Markine, V.L., 'Use of simplified numerical models as approximations: Application to a dynamical optimal design problem', In: ISSMO/NASA 
First Internet Conference on Approximation and Fast Reanalysis Techniques in Engineering Optimization, 1998

[10] Knill, D.L., Giunta, A.A., Baker, C.A., Grossman, B., Mason, W.H., Haftka, R.T., and Watson, L.T., 'Response Surface Methods Combining Linear and Euler Aerodynamics for Supersonic Transport Design', Journal of Aircraft, Vol. 36, No. 1, pp. 75-86, 1999.

[11] Kennedy, M.C., and O'Hagan, A., 'Predicting the Output from a Complex Computer Code When Fast Approximations Are Available', Biometrika, Vol.87, No.1, pp 1-13, 2000 .

[12] Huang, D., Allen, T.T., Notz, W.I., and Miller, R.A., 'Sequential Kriging Optimization Using Multiple-Fidelity Evaluations', Structural and Multidisciplinary Optimization, Vol. 32, No. 5, pp 369-382, 2006

[13] Forrester, A.I.J., Sobester, A., and Keane, A.J., 'Multi-Fidelity Optimization via Surrogate Modelling', Proceedings of the Royal Society of London, series A: Mathematical and Physical Sciences, Vol. 463, No. 2088, pp.3251-3269, 2007

[14] Leary, S.J., Bhaskar, A., and Keane, A.J., 'A Knowledge-Based Approach To response Surface Modelling in Multifidelity Optimization', Journal of Global Optimization, Vol. 26, No. 3, pp.297-319, 2003

[15] Robinson, T.D., Eldred, M.S., Willcox, K.E., Haimes, R., 'Strategies for multifidelity optimization with variable dimensional hierarchical models', In: $47^{\text {th }}$ AIAA/ASME/ASCE/AHS/ASC Structures, Structural Dynamics, and Materials Conference, Newport, Rhode Island, 2006

[16] Robinson, T.D., Willcox, K.E., Eldred, M.S., Haimes, R., 'Multifidelity optimization for variable-complexity design', In: 11th AIAA/ISSMO/ Multidisciplinary Analysis and Optimization Conference, Portsmouth, Virginia, 2006

[17] Everson, R., Sirovich, L., 'The Karhunen-Loeve procedure for gappy data', J. Opt. Soc. Am., 12: 1657-1664, 1995.

[18] Reisenthel, P.H., Love, J.F., Lesieutre, D.J. and Dillenius, M.F.E., 'Innovative Fusion of Experiment and Analysis for Missile Design and Flight Simulation', RTO Symposium on Innovative Missile Systems, Amsterdam, 15-18 May 2006, Report RTO-MP-AVT-135, 2006. 
[19] Rendall, T.C.S., and Allen, C.B., 'Multidimensional Aircraft Data Interpolation using Radial Basis Functions', 25 ${ }^{\text {th }}$ AIAA Applied Aerodynamics Conference, Miami, FL. June 2007.

[20] Mifsud M., 'Reduced-order modelling for high-speed aerial weapon aerodynamics', Ph.D. Thesis, Cranfield University, 2008.

[21] Ludlow D.K., 'IMPNS User's Manual', Cranfield University, CoA Report NFP-0113, 2001.

[22] Ludlow D.K., 'IMPNS Theory Guide', Cranfield Univesity, CoA Report NFP-0112, 2001.

[23] Qin N., Richards B.E., 'Finite volume 3DNS and PNS solutions of hypersonic viscous flows around a delta wing using Osher's flux difference splitting', Proceedings of a Workshop on Hypersonic Flows for Re-entry Problems, 1990.

[24] Qin N, Jayatunga C., 'Algebraic turbulence modelling for vortical flows around slender Bodies', NATO RTO-MP-5, Missile Aerodynamics, Paper 20, 1998.

[25] Shaw S., Qin N., 'A matrix-free preconditioned Krylov subspace method for the PNS Equations', AIAA 98-111, 1998.

[26] Qin N., Ludlow D.K., 'A cure for anomalies of Osher and AUSM+ schemes for hypersonic viscous flows around swept cylinders', Proceedings of the 22nd International Symposium on Shock Waves, Imperial College, London, UK, 1999; 635-640 (Editors: Ball GJ, Hillier $\mathrm{R}$ and Roberts GT)

[27] Qin N., Ludlow D.K., Zhong B., Shaw S.T., Birch T.J., 'Multigrid acceleration of a preconditioned GMRES implicit PNS solver', AIAA 99-0779, 1999.

[28] Shaw S., Mifsud M., Wagner B., Moureaux A., Bennett J., 'IMPNS Computations of the X-15 Flight Vehicle: A Validation and Verification Case Study', NAFEMS International Journal of CFD Case Studies Vol. 6, March 2007.

[29] Mifsud M., Estruch-Samper D., MacManus D., Chaplin R., Stollery J., 'A case study on the aerodynamic heating of a hypersonic vehicle', The Aeronautical Journal, Vol. 116 Paper No. 1183, September 2012.

[30] Lumley J.L., 'The structure of inhomogenous turbulence. Atmospheric Turbulence and Wave Propagation', Nauka, Moscow, 1967; 166-178 (Editors: Yaglom AM, Tatarski VI.)

[31] Epureanu BI, Dowell EH, Hall K. A Parametric Analysis of Reduced Order Models of Potential Flows in Turbomachinery Using Proper Orthogonal Decomposition. 2001-GT- 
0434, Proceedings of ASME TURBO EXPO 2001, New Orleans, Louisiana, 2001.

[32] LeGresley P, Alonso J. Investigation of Non-Linear Projection for POD based Reduced Order Models for Aerodynamics. AIAA 2001-0926, 39 ${ }^{\text {th }}$ Aerospace Sciences Meeting and Exhibit, Reno, NV, 2001.

[33] Bui-Thanh T., Damodaran M., Willcox K., 'Proper Orthogonal Decomposition Extensions for Parametric Applications in Compressible Aerodynamics', AIAA 2003-4213, $21^{\text {st }}$ Applied Aerodynamics Conference, Orlando, FL, 2003.

[34] Mifsud M.J., Shaw S.T., MacManus, D.G., 'A high-fidelity low-cost aerodynamic model using proper orthogonal decomposition', Inter. Journal for Numerical Methods in Fluids 2009.

[35] Kirby M., Boris J.P., Sirovich L., 'A Proper Orthogonal Decomposition of a Simulated Supersonic Shear Layer', Inter. Journal for Numerical Methods in Fluids 1990; 10: 411 428.

[36] Buhmann M.D., 'Radial Basis Functions: Theory and Implementations', Cambridge Monographs on Applied and Computational Mathematics, 2003.

[37] Kansa E.J., 'Multiquadrics - A scattered data approximation scheme with applications to computational fluid-dynamics - Surface Approximations and Partial Derivative Estimates', Computers Math. Applic. 1990; 19 (8/9): 127-145.

[38] Carlson R.E., Foley T.A., 'The Parameter $\mathrm{R}^{2}$ in Multiquadric Interpolation', Computers Math. Applic. 1991; 21(9): 29-42. 


\begin{tabular}{|c|c|c|c|c|c|c|c|}
\hline \multirow{2}{*}{$\begin{array}{c}\text { Angle } \\
\text { of } \\
\text { attack }\end{array}$} & $\begin{array}{c}\text { Surface } \\
\text { Parameter }\end{array}$ & $\begin{array}{c}\text { Correlation } \\
\text { Coefficient }\end{array}$ & $\begin{array}{c}\text { Lower } \\
\text { Limit }\end{array}$ & $\begin{array}{c}\text { Upper } \\
\text { Limit }\end{array}$ & $\begin{array}{c}\text { Correlation } \\
\text { Coefficient }\end{array}$ & $\begin{array}{c}\text { Lower } \\
\text { Limit }\end{array}$ & $\begin{array}{c}\text { Upper } \\
\text { Limit }\end{array}$ \\
\cline { 2 - 8 } & $\mathbf{C}_{\mathbf{p}}$ & 0.9888 & 0.9872 & 0.9903 & 0.9950 & 0.9943 & 0.9957 \\
\cline { 2 - 8 } $\mathbf{0 . 0}^{\circ}$ & $\boldsymbol{\tau}_{\mathbf{x}}$ & 0.9606 & 0.9549 & 0.9656 & 0.8011 & 0.7749 & 0.8245 \\
\cline { 2 - 8 } & $\boldsymbol{\tau}_{\mathbf{y}}$ & 0.9756 & 0.9720 & 0.9787 & 0.8374 & 0.8155 & 0.8569 \\
\cline { 2 - 8 } & $\boldsymbol{\tau}_{\mathbf{z}}$ & 0.9729 & 0.9690 & 0.9764 & 0.8689 & 0.8509 & 0.8849 \\
\hline \multirow{4}{*}{$\mathbf{2 . 0}^{\circ}$} & $\mathbf{C}_{\mathbf{p}}$ & 0.9886 & 0.9869 & 0.9901 & 0.9935 & 0.9926 & 0.9944 \\
\cline { 2 - 8 } & $\boldsymbol{\tau}_{\mathbf{x}}$ & 0.9372 & 0.9283 & 0.9451 & 0.8017 & 0.7756 & 0.8251 \\
\cline { 2 - 8 } & $\boldsymbol{\tau}_{\mathbf{y}}$ & 0.9474 & 0.9398 & 0.9540 & 0.8639 & 0.8452 & 0.8804 \\
\cline { 2 - 8 } & $\boldsymbol{\tau}_{\mathbf{z}}$ & 0.9479 & 0.9404 & 0.9545 & 0.8918 & 0.8767 & 0.9051 \\
\hline \multirow{4}{*}{$\mathbf{5 . 0}^{\circ}$} & $\mathbf{C}_{\mathbf{p}}$ & 0.9872 & 0.9854 & 0.9889 & 0.9937 & 0.9928 & 0.9945 \\
\cline { 2 - 8 } & $\boldsymbol{\tau}_{\mathbf{x}}$ & 0.9268 & 0.9164 & 0.9360 & 0.9153 & 0.9034 & 0.9259 \\
\cline { 2 - 8 } & $\boldsymbol{\tau}_{\mathbf{y}}$ & 0.9156 & 0.9037 & 0.9261 & 0.8994 & 0.8853 & 0.9118 \\
\cline { 2 - 8 } & $\boldsymbol{\tau}_{\mathbf{z}}$ & 0.9141 & 0.9020 & 0.9248 & 0.9115 & 0.8991 & 0.9225 \\
\hline \multirow{3}{*}{$\mathbf{8 . 0}^{\circ}$} & $\mathbf{C}_{\mathbf{p}}$ & 0.9863 & 0.9843 & 0.9881 & 0.9962 & 0.9956 & 0.9967 \\
\cline { 2 - 8 } & $\boldsymbol{\tau}_{\mathbf{x}}$ & 0.9593 & 0.9534 & 0.9644 & 0.9520 & 0.9451 & 0.9581 \\
\cline { 2 - 8 } & $\boldsymbol{\tau}_{\mathbf{y}}$ & 0.9533 & 0.9466 & 0.9592 & 0.9344 & 0.9250 & 0.9426 \\
\cline { 2 - 8 } & $\boldsymbol{\tau}_{\mathbf{z}}$ & 0.9517 & 0.9447 & 0.9578 & 0.9385 & 0.9298 & 0.9463 \\
\hline \multirow{3}{*}{$\mathbf{1 0 . 0}^{\circ}$} & $\mathbf{C}_{\mathbf{p}}$ & 0.9863 & 0.9842 & 0.9880 & 0.9978 & 0.9975 & 0.9981 \\
\cline { 2 - 8 } & $\boldsymbol{\tau}_{\mathbf{x}}$ & 0.9793 & 0.9762 & 0.9819 & 0.9700 & 0.9657 & 0.9739 \\
\cline { 2 - 8 } & $\boldsymbol{\tau}_{\mathbf{y}}$ & 0.9803 & 0.9774 & 0.9828 & 0.9639 & 0.9586 & 0.9685 \\
\cline { 2 - 8 } & $\boldsymbol{\tau}_{\mathbf{z}}$ & 0.9806 & 0.9778 & 0.9831 & 0.9635 & 0.9582 & 0.9681 \\
\hline
\end{tabular}

Table 1 - Pearson's product-moment correlation coefficient evaluated upon the surface parameters at a Mach number of 2.0 and 6.0 between the low- and high-fidelity snapshots for the conecylinder-flare body 


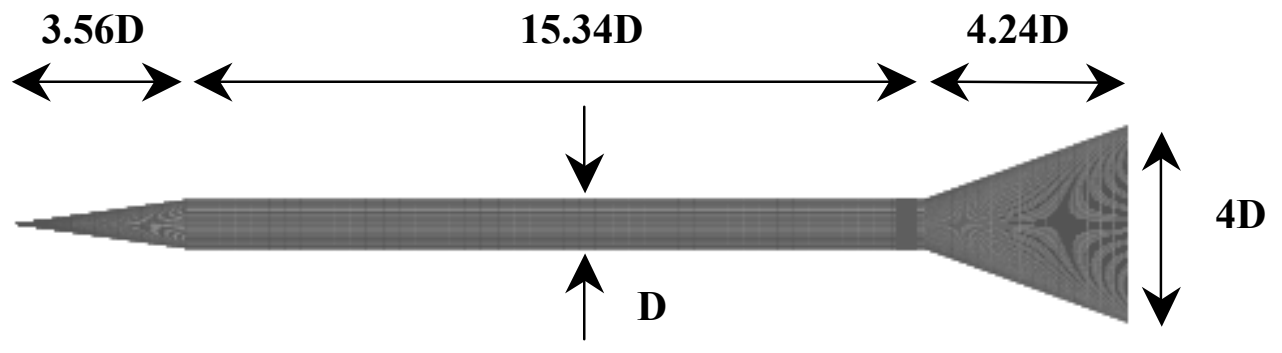

Figure 1 - Body geometry of total length 23.14D where D is the mid-section diameter 


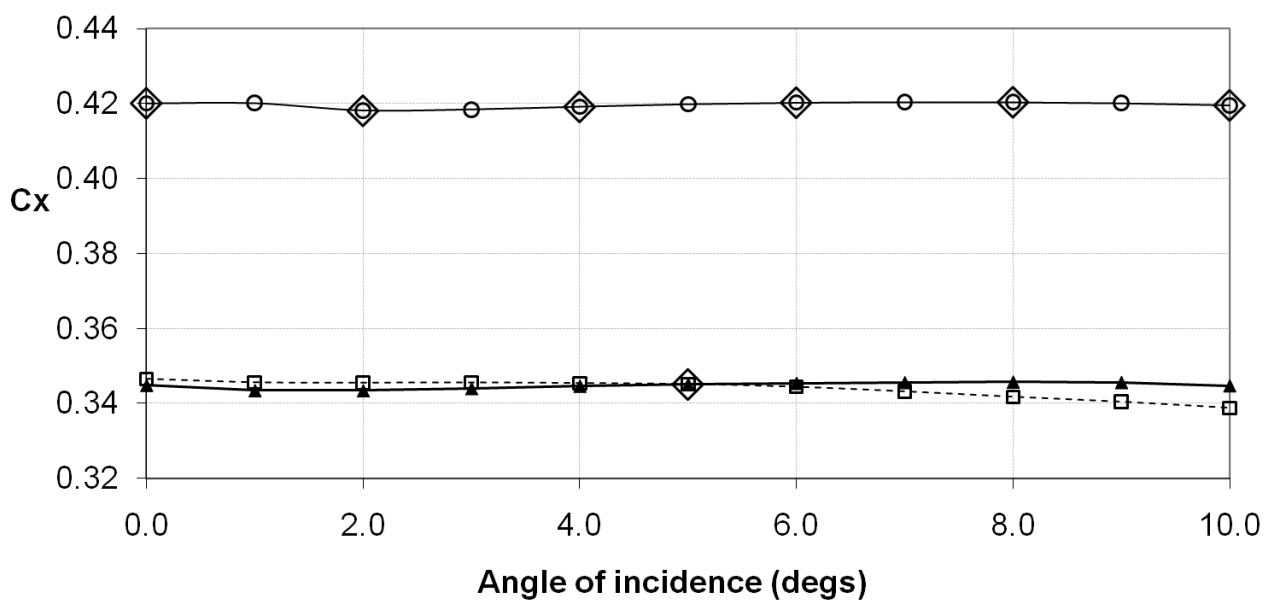

- - - High Order Accurate $\quad-$-First Order Accurate $\rightarrow$ Prediction $\diamond$ Training Data

(a) $\mathbf{C}_{\mathbf{x}}$

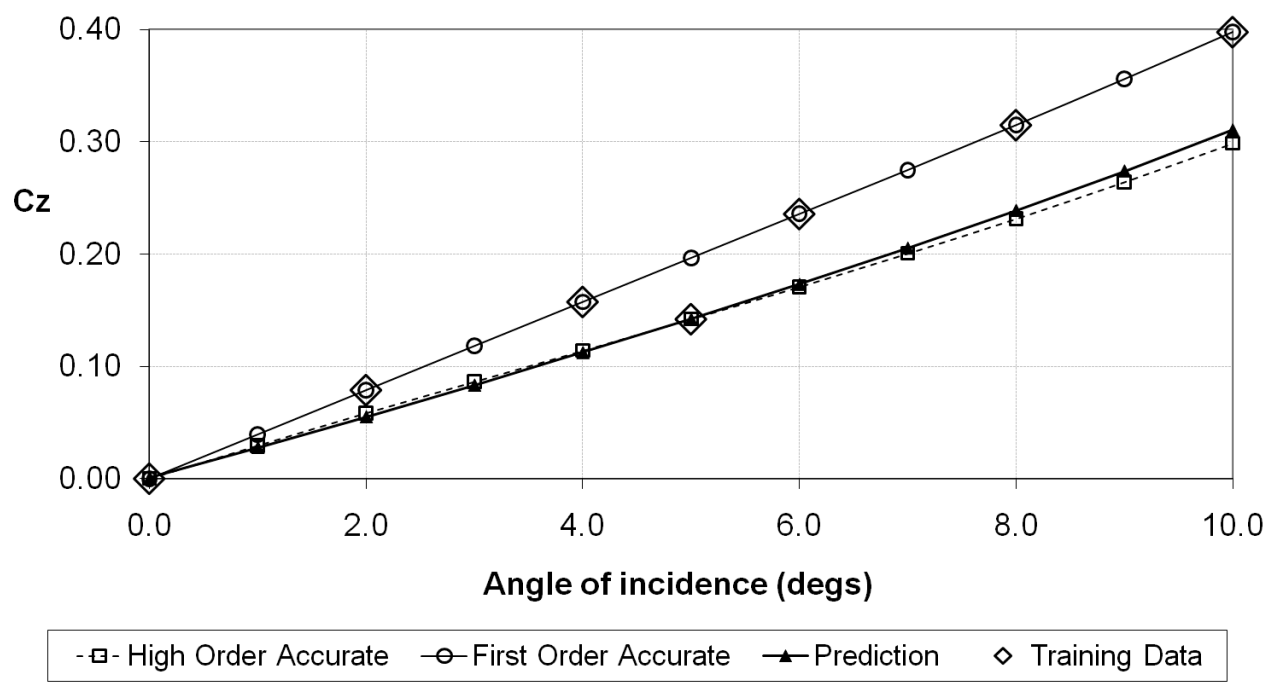

(b) $\mathbf{C}_{\mathrm{z}}$

Figure 2 - Calculations and predictions for $C_{x}$ and $C_{z}$ as a function of angle of incidence using 5 high-fidelity snapshots for $M=2$ and $R e=0.4$ million 


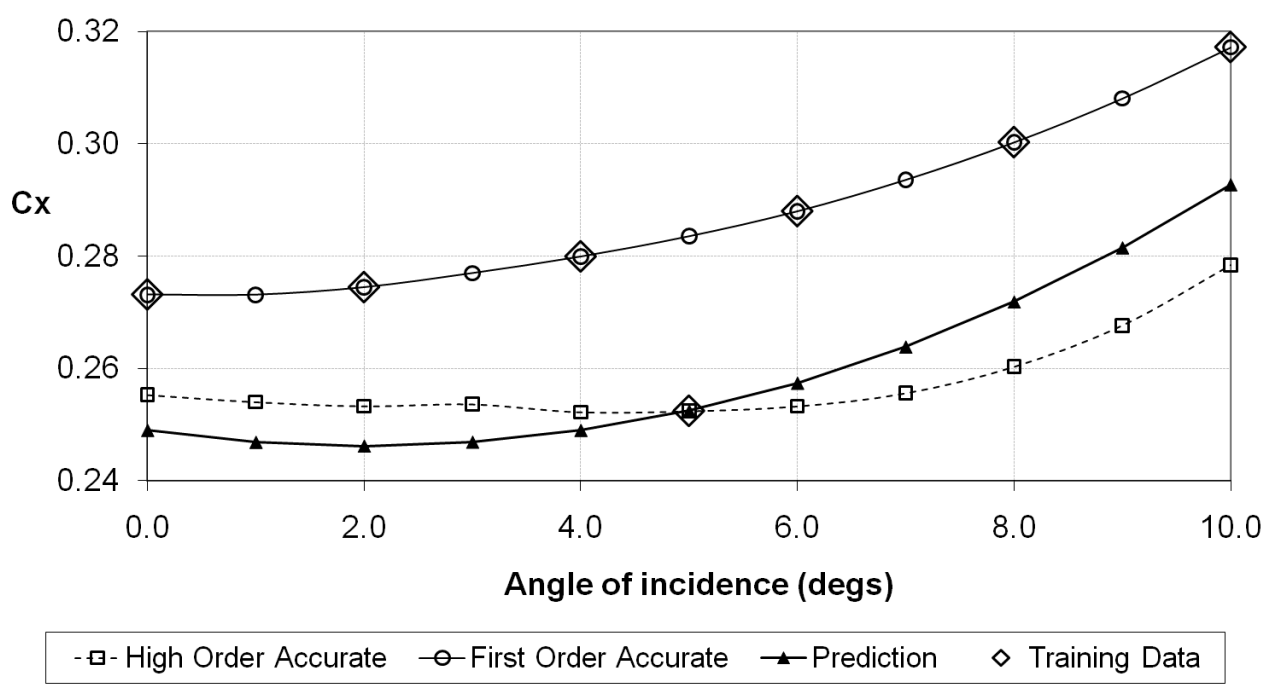

(a) $\mathbf{C}_{\mathrm{x}}$

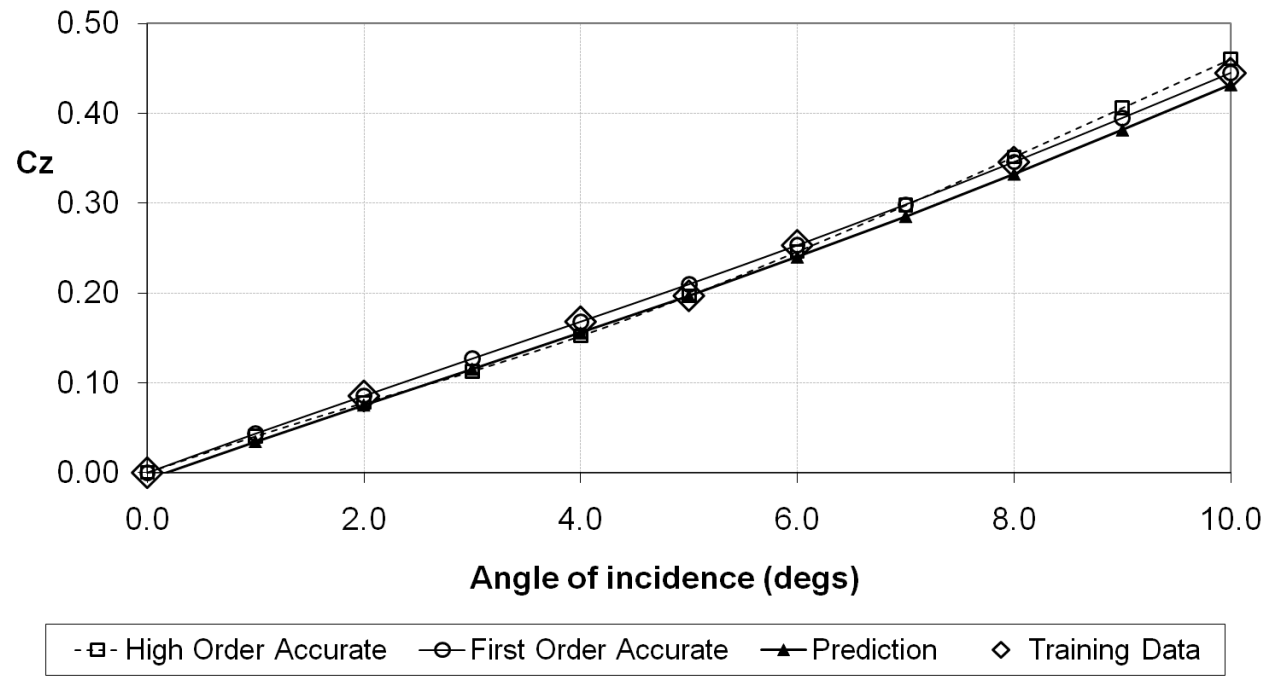

(b) $\mathbf{C}_{\mathrm{z}}$

Figure 3 - Calculations and predictions for $C_{x}$ and $C_{z}$ as a function of angle of incidence using 5 high-fidelity snapshots for $M=6$ and $R e=0.4$ million 


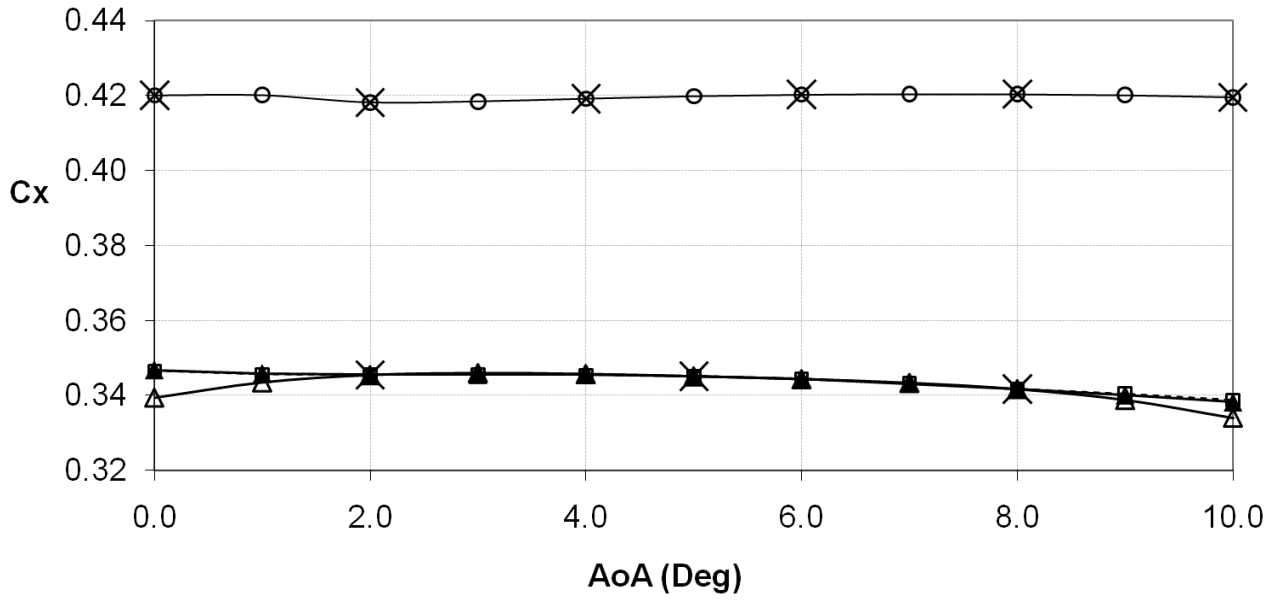

- $\square-\mathrm{Hi}-\mathrm{Fi} \quad-$ Lo-Fi $\rightarrow$ Prediction $\triangle$ Prediction V-S only $\times$ Training Data

(a) $\mathbf{C}_{\mathrm{x}}$

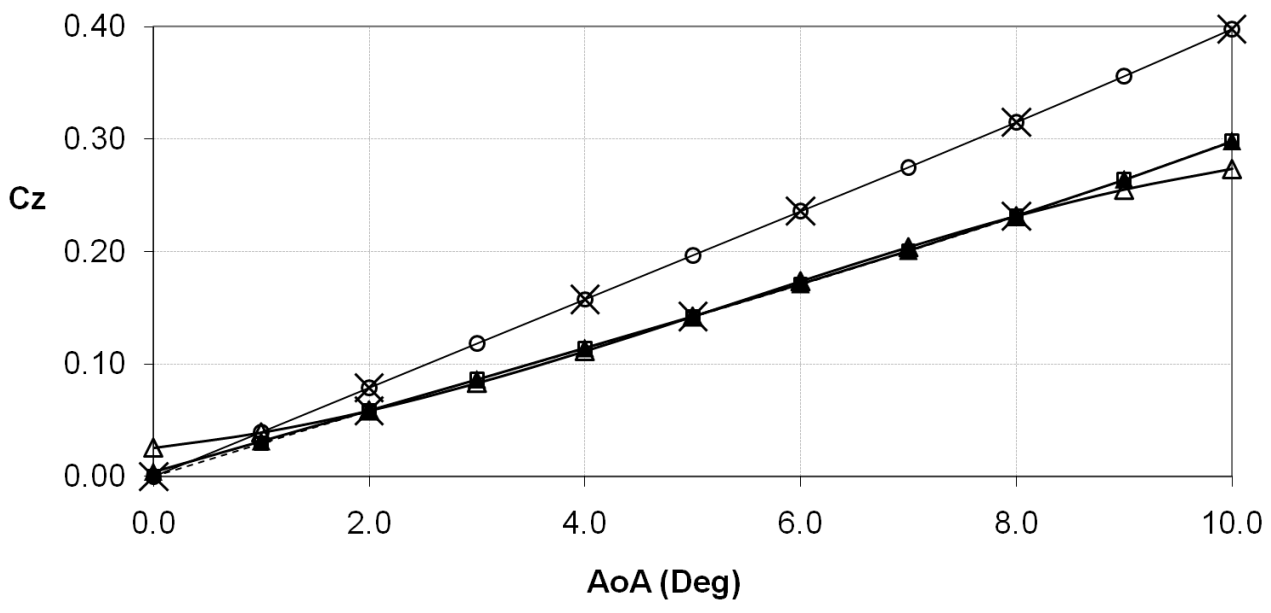

๑- Hi-Fi $\quad-$ Lo-Fi $\rightarrow$ Prediction $\triangle$ Prediction V-S only $\times$ Training Data

(b) $\mathbf{C}_{\mathbf{z}}$

Figure 4 - Calculations and predictions for $C_{x}$ and $C_{z}$ as a function of angle of incidence using 15 high-fidelity snapshots for $M=2$ and $R e=0.4$ million 


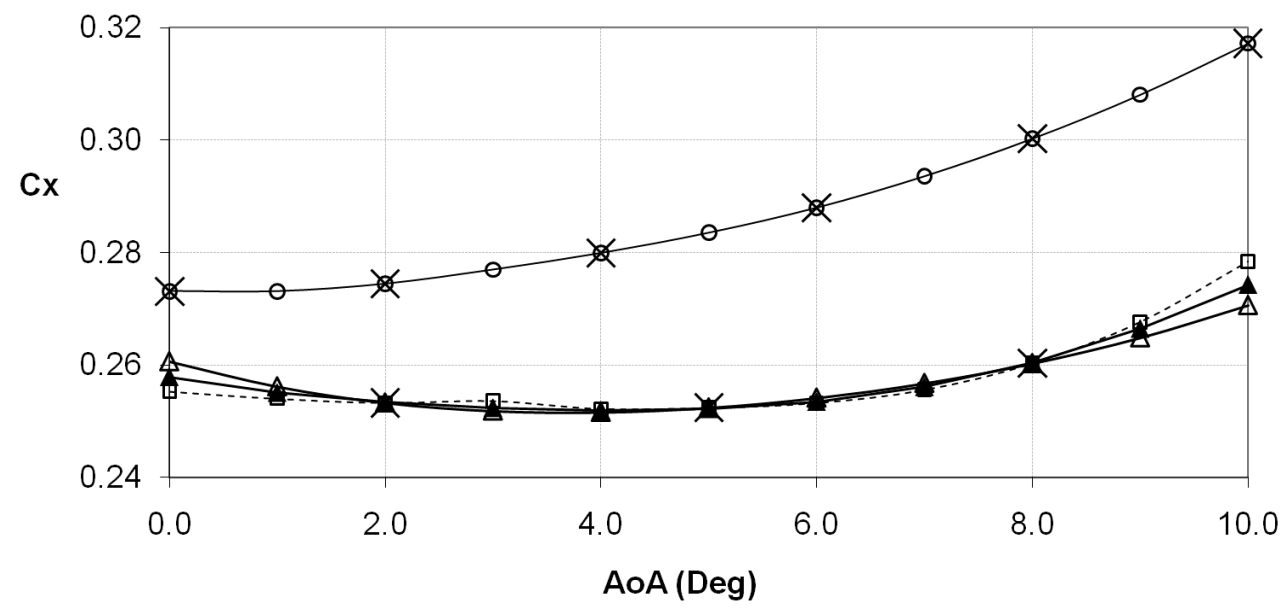

- $\square$ - Hi-Fi $\quad-$ - Lo-Fi $\rightarrow$-Prediction $\triangle$-Prediction V-S only $\times$ Training Data

(a) $\mathrm{C}_{\mathbf{x}}$

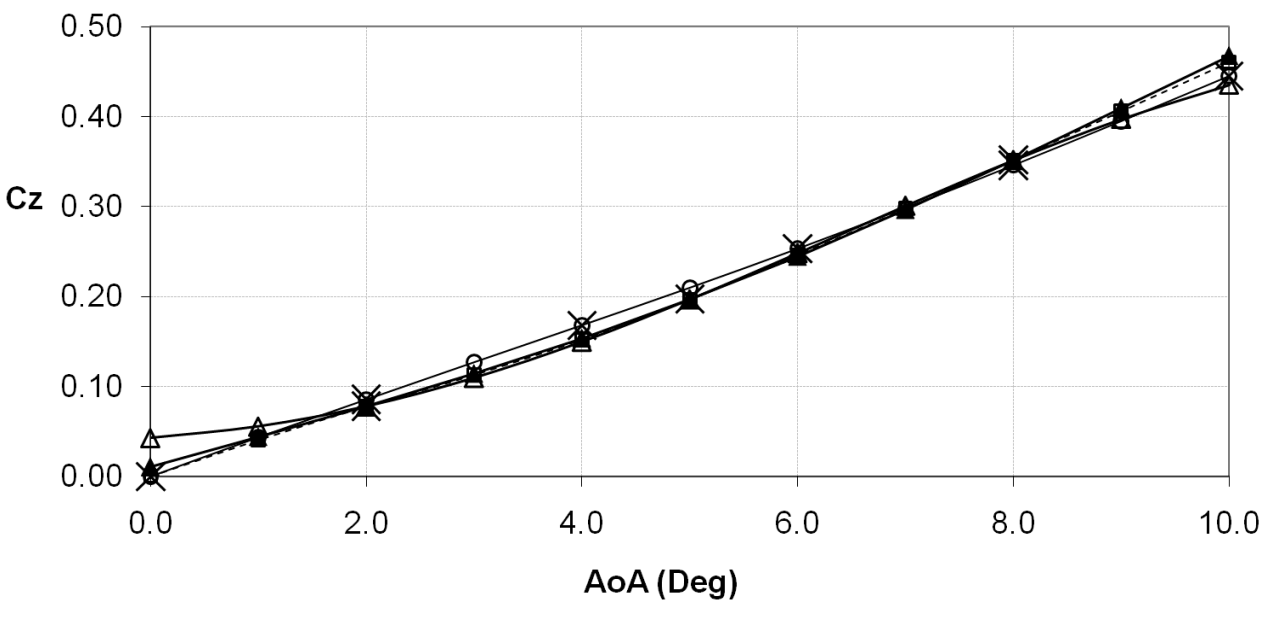

๑- Hi-Fi $\quad-$ Lo-Fi $\rightarrow$ Prediction $\triangle$ Prediction V-S only $\times$ Training Data

(b) $\mathrm{C}_{\mathrm{z}}$

Figure 5 - Calculations and predictions for $C_{x}$ and $C_{z}$ as a function of the angle of incidence using 15 high-fidelity snapshots for $M=6$ and $R e=0.4$ million 


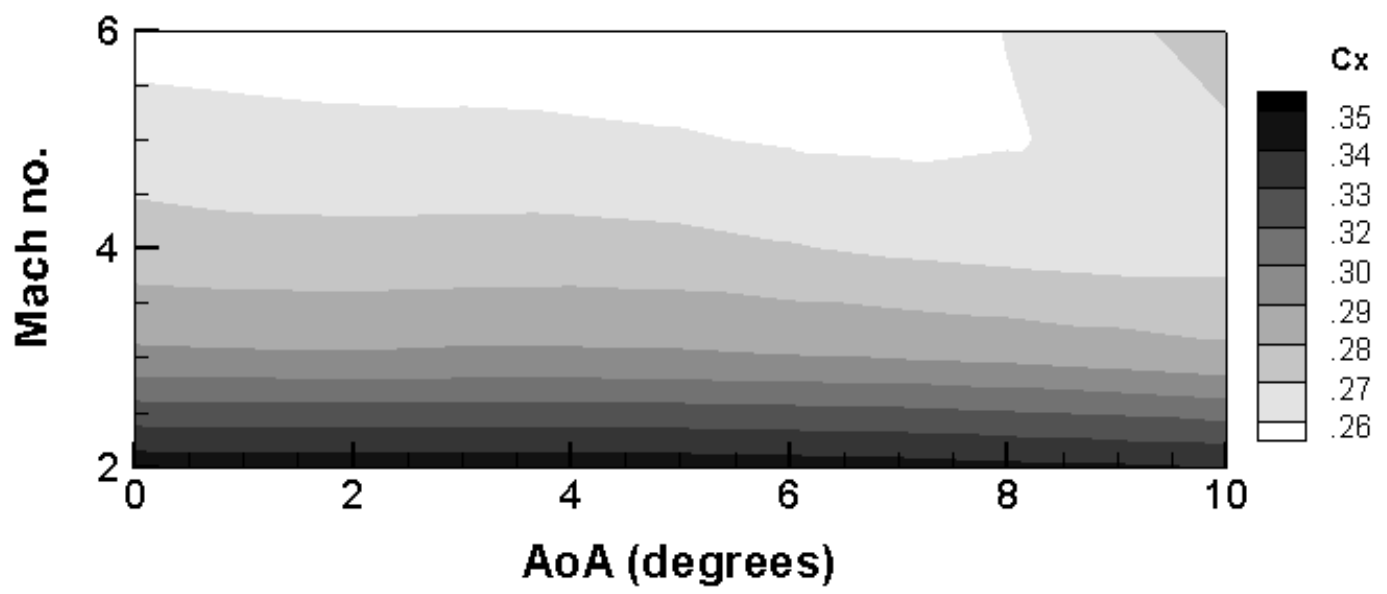

(a) using 55 high-fidelity CFD solutions

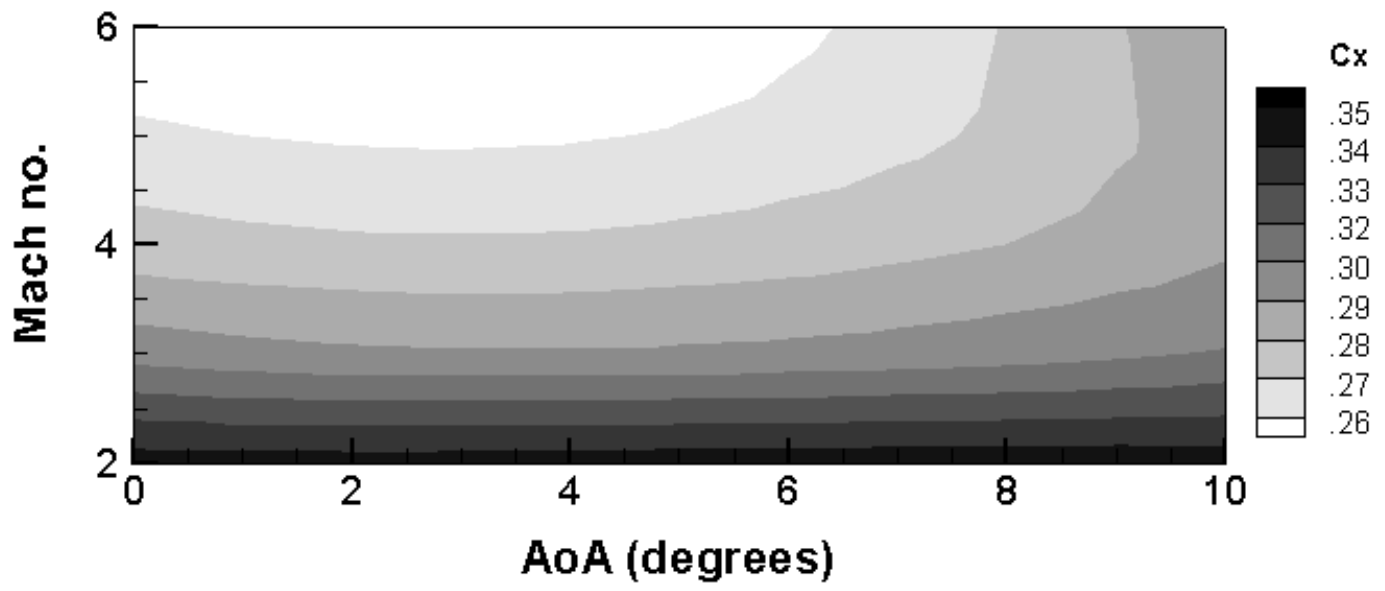

(b) predicted from the variable-fidelity model based on POD with 5 high-fidelity snapshots

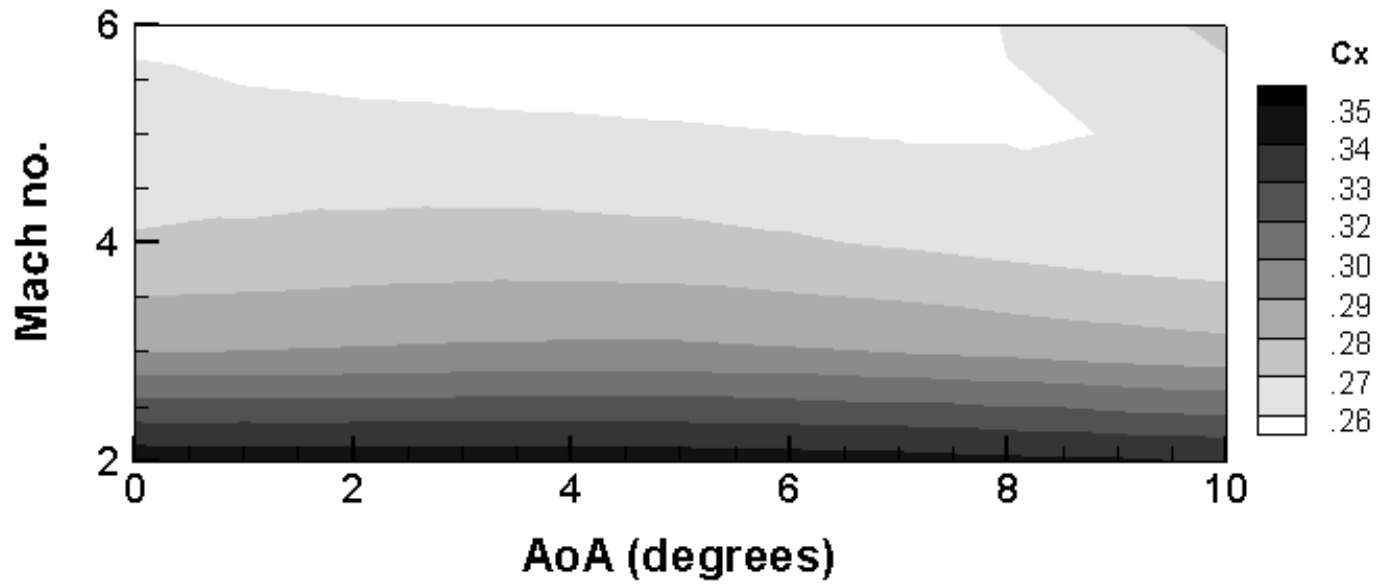

(c) predicted from the variable-fidelity model based on POD with 15 high-fidelity snapshots

Figure 6 - Projected carpet plots of the viscous axial force coefficient $C_{x}$ 


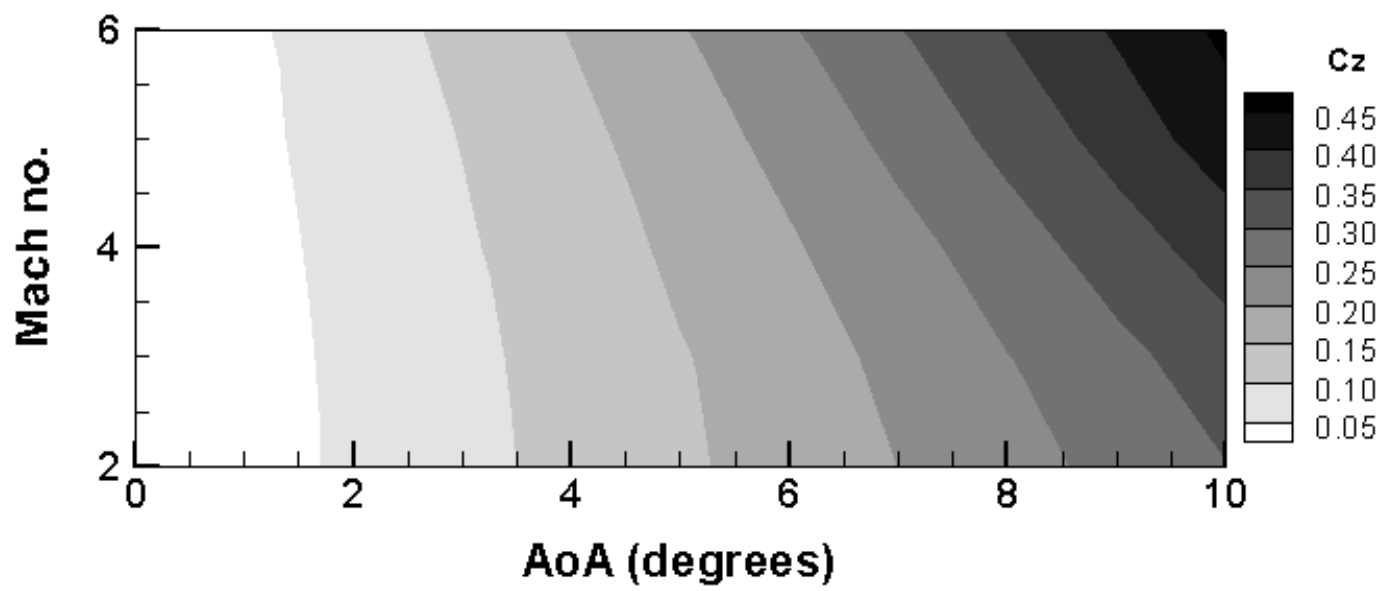

(a) using 55 high-fidelity CFD solutions

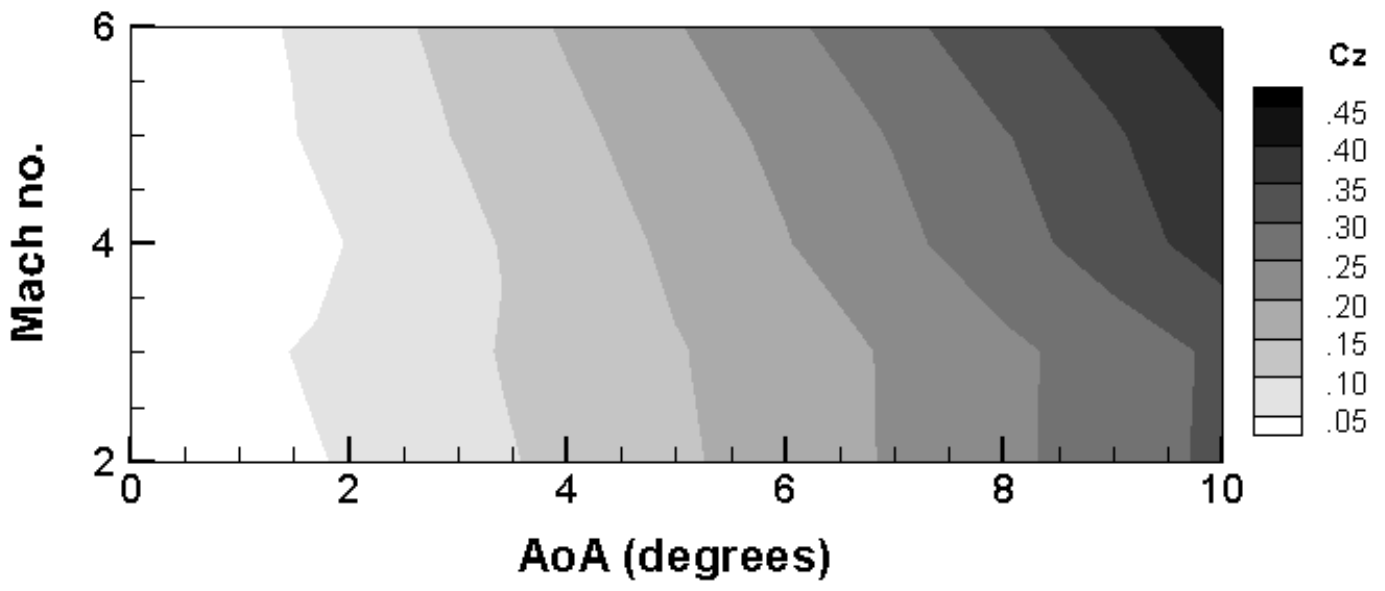

(b) predicted from the variable-fidelity model based on POD with 5 high-fidelity snapshots

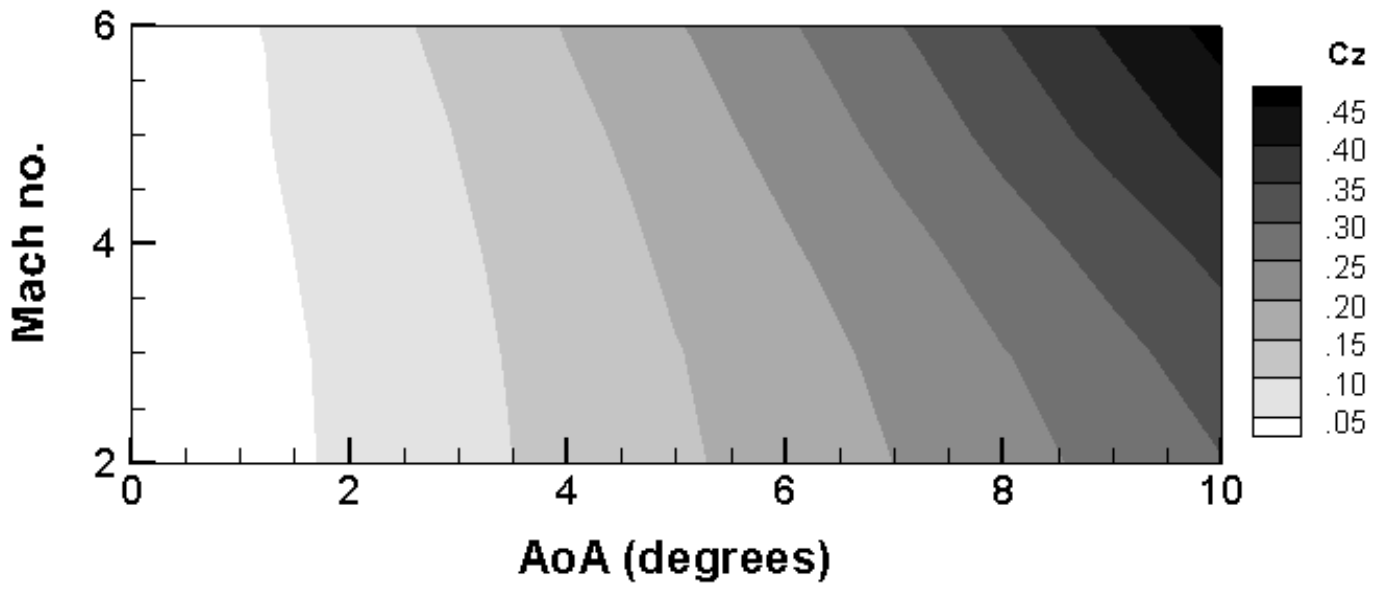

(c) predicted from the variable-fidelity model based on POD with 15 hi-fi snapshots

Figure 7 - Projected carpet plots of the normal force coefficient $C_{z}$ 


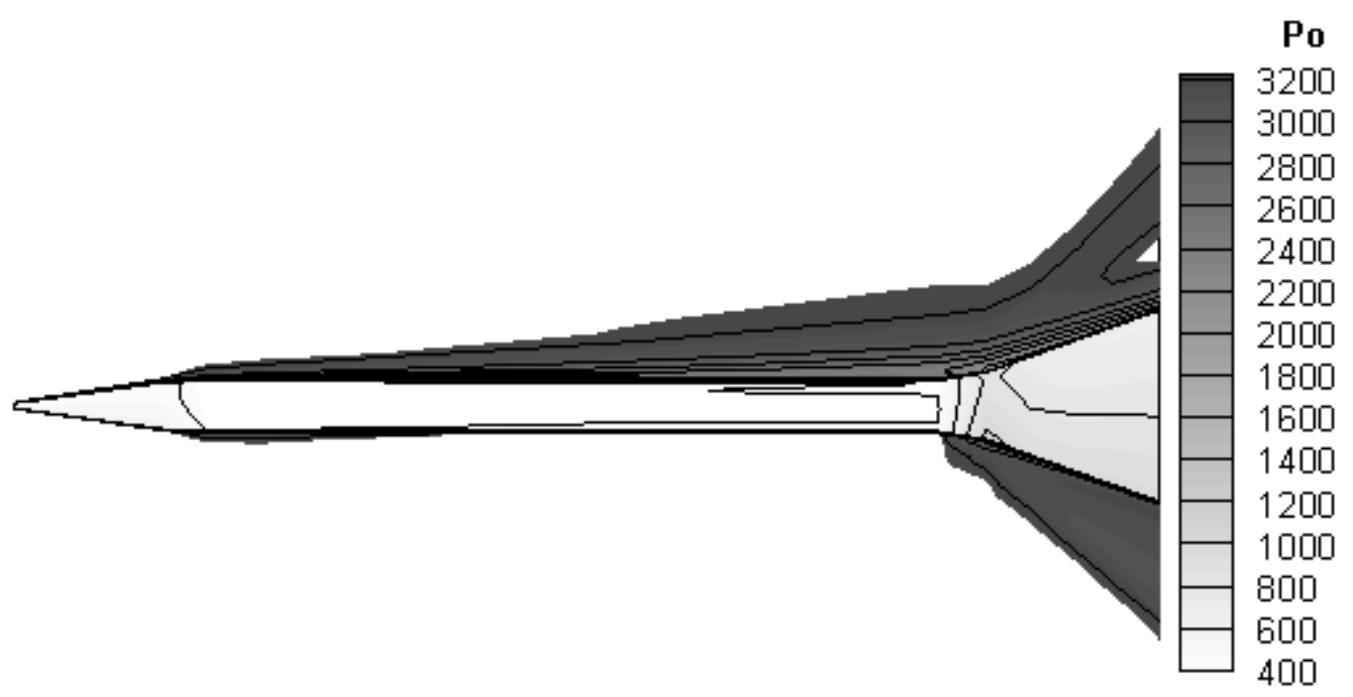

(a)

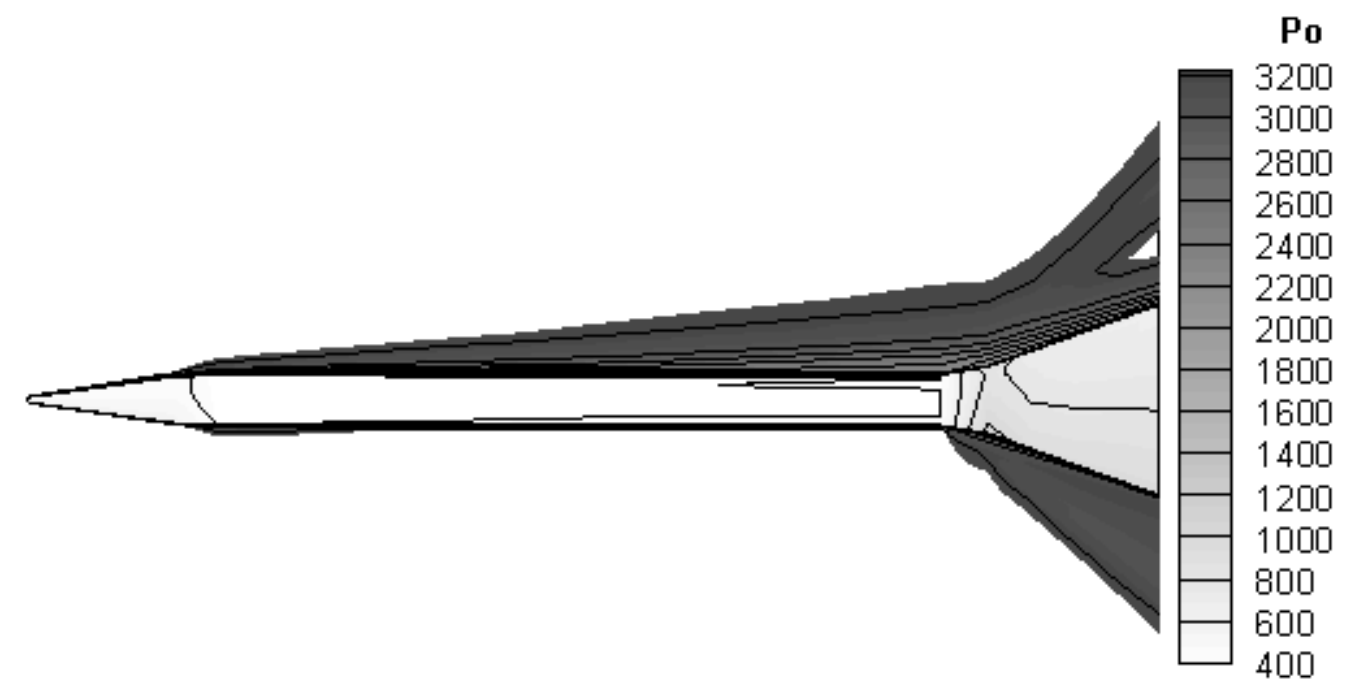

(b)

Figure 8 - Comparison of the total pressure contours at $M=2$ and alpha $=6^{\circ}$ (a) VFM predicted total pressure contours (b) High-fidelity CFD simulation total pressure contours 


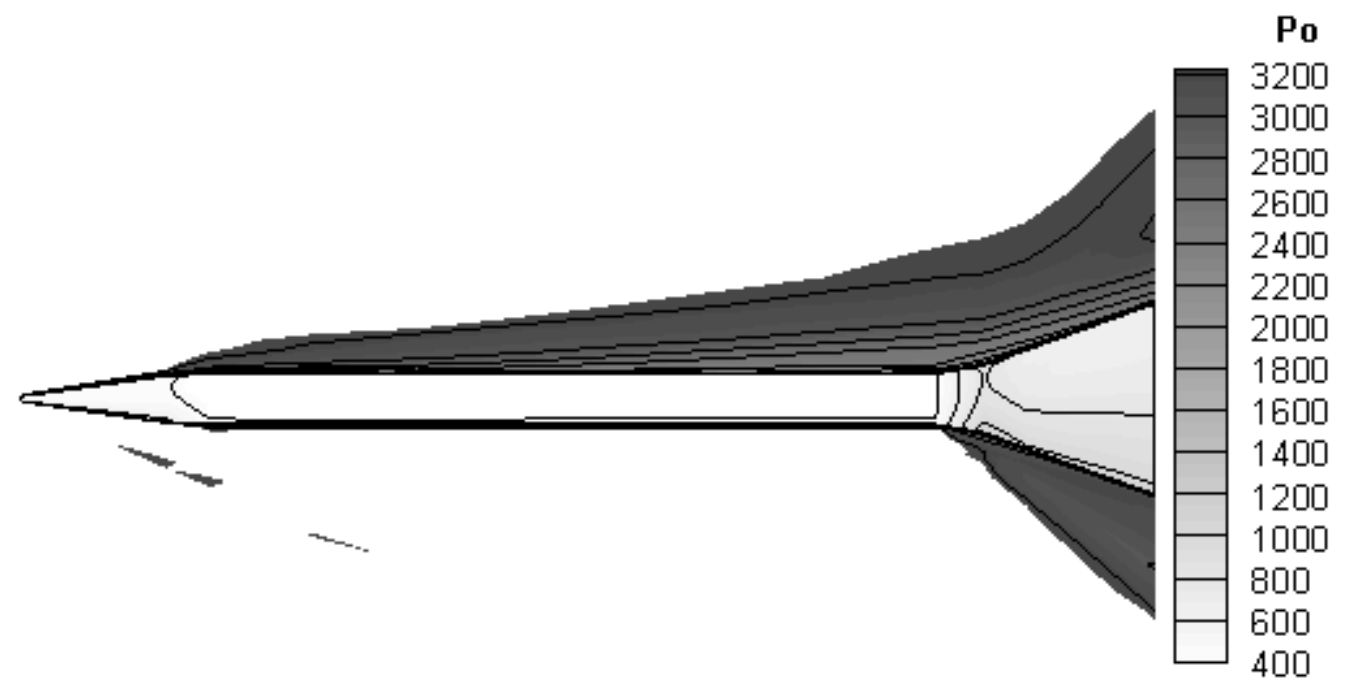

(a)

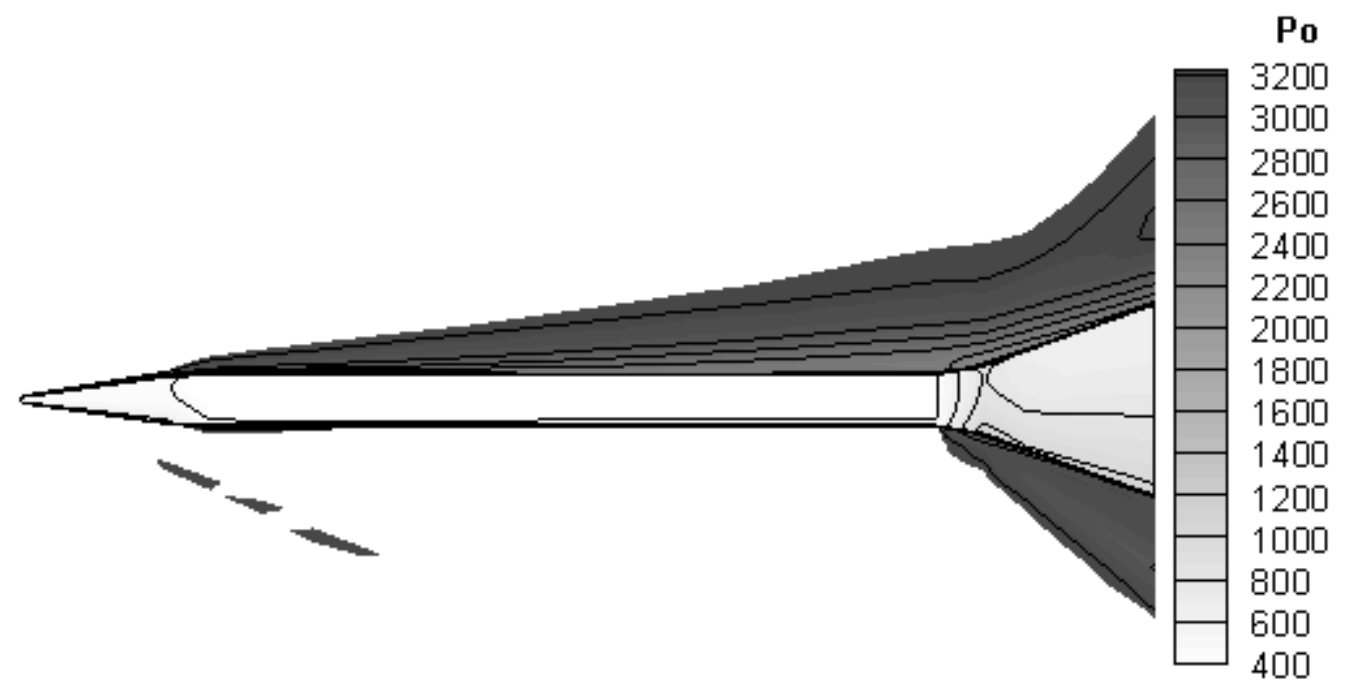

(b)

Figure 9 - Comparison of the total pressure contours at $M=2$ and alpha $=9^{\circ}$ (a) VFM predicted total pressure contours (b) High-fidelity CFD simulation total pressure contours 


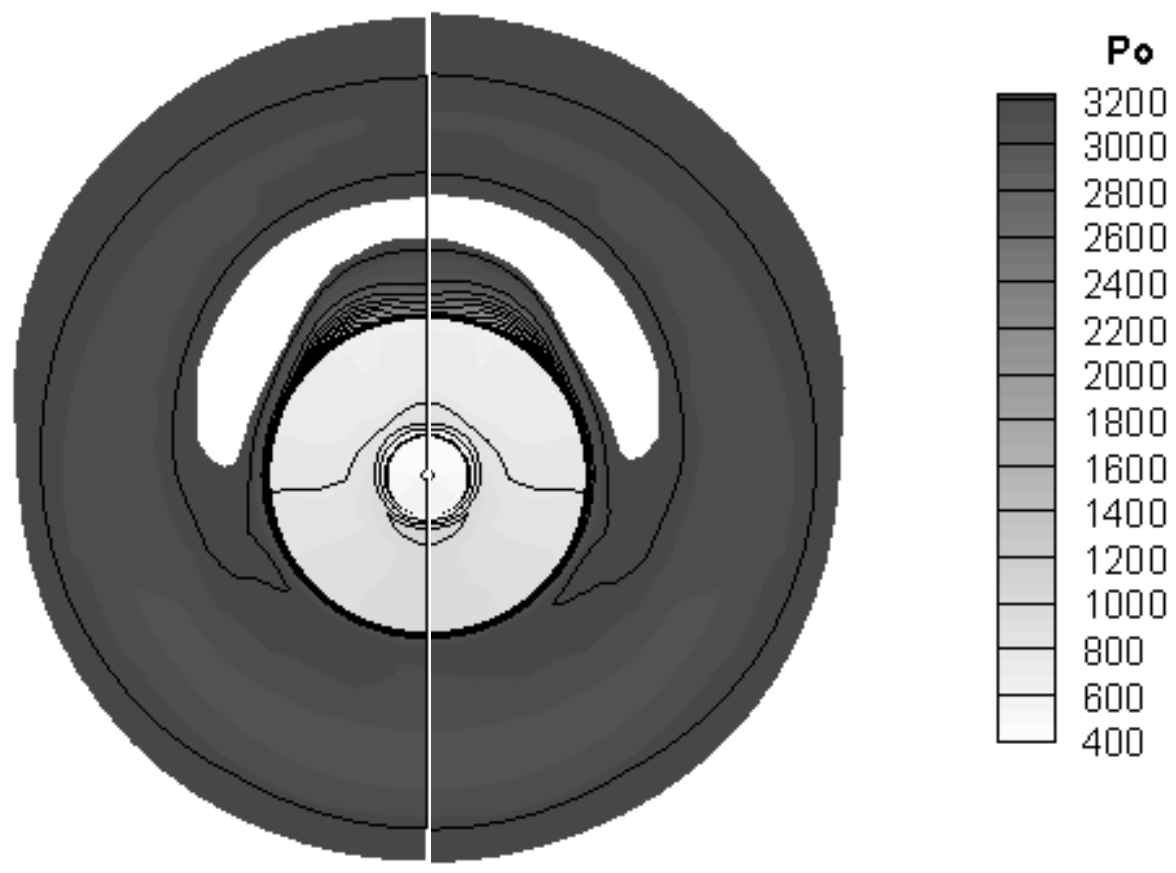

Figure 10 - Cross-sectional view of the total pressure contours at $M=2$ and alpha $=6^{\circ}$ (a) VFM predicted total pressure contours on the LHS (b) High-fidelity CFD simulation total pressure contours on the RHS 


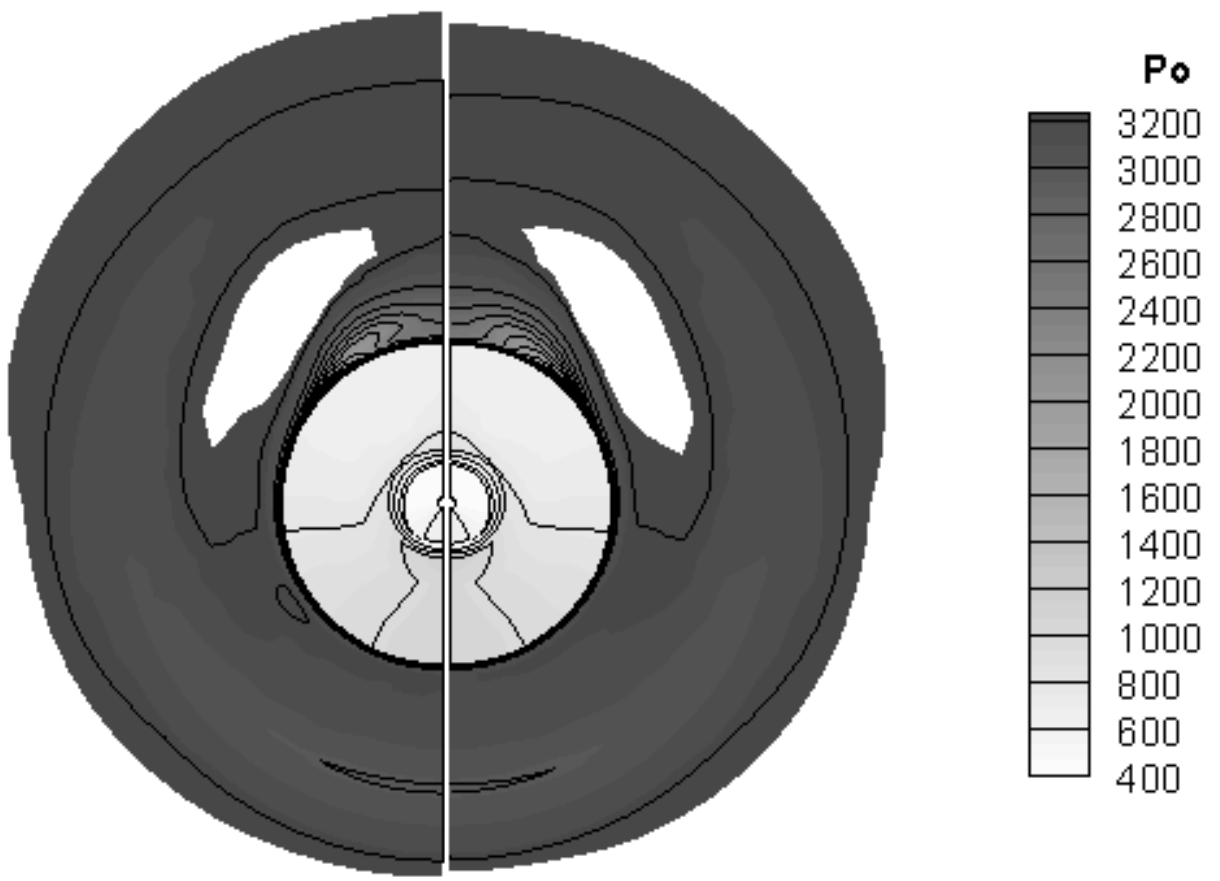

Figure 11 - Cross-sectional view of the total pressure contours at $M=2$ and alpha $=9^{\circ}$ (a) VFM predicted total pressure contours on the LHS (b) High-fidelity CFD simulation total pressure contours on the RHS 
2016-04-20

\section{A variable-fidelity aerodynamic model using proper orthogonal decomposition}

Mifsud, Michael

Wiley

Mifsud MJ, MacManus DG, Shaw ST, A variable-fidelity aerodynamic model using proper orthogonal decomposition, International Journal for Numerical Methods in Fluids, Volume 82 , pÿlssue 10, 10 December 2016, Pages 646663 http://dx.doi.org/10.1002/fld.4234

Downloaded from Cranfield Library Services E-Repository 